Miryuta, N., Parnikoza, I., Poronnik, O., Myryuta, G., Rojek-

Jelonek, M., Dykyi, E., Kunakh, V. (2021). Calculation of United

Quality Latent Indices of Deschampsia antarctica plants adaptability

of different origin grown in vitro.

Ukrainian Antarctic Journal, 1, 56-81.

https://doi.org/10.33275/1727-7485.1.2021.667
Біологічні дослідження

Biological Research

N. Miryuta ${ }^{\text {, I. Parnikoza 1, 2,3, *, O. Poronnik }}{ }^{2,1}$, G. Myryuta ${ }^{2,1}$, M. Rojek-Jelonek ${ }^{4}$, E. Dykyi ${ }^{1}$, V. Kunakh ${ }^{2}$

${ }^{1}$ State Institution National Antarctic Scientific Center, Ministry of Education and Science of Ukraine, Kyiv, 01601, Ukraine

${ }^{2}$ Institute of Molecular Biology and Genetics, National Academy of Sciences of Ukraine, Kyiv, 03143, Ukraine

${ }^{3}$ National University of "Kyiv-Mohyla Academy", Kyiv, 04655, Ukraine

${ }^{4}$ University of Silesia in Katowice, Katowice, 40-032, Poland

*Corresponding author: ivan.parnikoza@uac.gov.ua

\title{
Calculation of United Quality Latent Indices of Deschampsia antarctica plants adaptability of different origin grown in vitro
}

\begin{abstract}
The research was to develop and describe in detail the algorithm for calculating the United Quality Latent Index

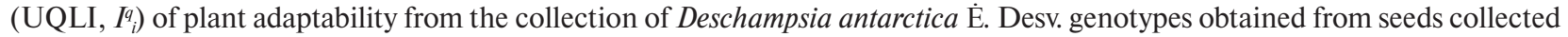
at different sites in the Argentine Islands region, the maritime Antarctic, and grown in vitro at the laboratory conditions. Genome size and genetic distances by ISSR and IRAP markers according to data from published articles were used as basic indices of initial genetic heterogeneity for analyzed plant genotypes. To assess individual adaptability indices for eleven $D$. antarctica genotypes, we used measurement of the leaf length morphometric index and determination of the flavonoids content by rutin and the content of photosynthetic pigments. The spectra of reserve and protective proteins in leaves were investigated by polyacrylamide gel electrophoresis. To obtain the United Quality Latent Index of Adaptability ( $I_{i}^{q}$, UQLI), the method of extreme grouping was used. The estimation of $I_{i}^{q}$ (UQLI) was performed using pairwise comparisons of indices from differences sets for each pair of genotypes. We developed and described in detail the algorithm for $I_{i}^{q}$ estimation for eleven $D$. antarctica genotypes. As an example of application, correlation models of probability relations of the indices are presented. To evaluate the complex adaptability for eleven $D$. antarctica genotypes grown in vitro we used developed algorithm for the UQLI calculation. The individuality of the adaptive portrait for all studied genotypes under in vitro cultivation conditions was shown. The influence of basic genetic characteristics (genome size and genetic distances) on auxin metabolism-related indices of leaf length and flavonoid content was shown. Such effect may be carried out by genetic characteristics both individually and together, probably via auxin metabolism. Among the eight genotypes researched, we distinguish four different variants by correlation models and two (positive and negative) by the general $I_{i}^{q}$ value. Thus the $I_{i}^{q}(\mathrm{UQLI})$ is proposed to describe a large number of source data at different organization levels which characterize sample genotypes by reducing the dimensions to one dimensionless number. This genotypes' individuality and the peculiarities of their grouping by $I_{i}^{q}$ should be taken into account when doing experimental studies using these genotypes as model plants, especially in experiments studying the regulation of productivity and the effect of the various exogenous factors, etc.
\end{abstract}

Keywords: Deschampsia antarctica, in vitro plant culture, the United Quality Latent Index of plant adaptability, probabilistic relation correlation models of different indices 


\section{Introduction}

Plants, while adapting to adverse external conditions, have developed a number of adaptation mechanisms at the levels of molecular, cellular, and physiological responses, such as stress protein production, increased antioxidant synthesis, and accumulation of compatible solutes (Kreps et al., 2002; Iordachescu \& Imai, 2008; Gill \& Tuteja, 2010; Lee et al., 2012). To take into account biodiversity and sometimes different directionality of adaptation mechanisms, the application of methods of their complex consideration within the integral index proved to be an urgent task.

In previous work, we have developed a methodology for assessing a complex index of adaptability based on individual adaptation indices to environmental conditions sets (Miryuta et al., 2019a; 2019b). We used the vascular plant Deschampsia antarctica $\dot{\mathrm{E}}$. Desv., 1854, which lives in one of the most extreme regions of the Earth - the Antarctic, because this plant species was able to adapt to various abiotic stressors through a plant reactions-coordinated response (Shinozaki et al., 2003; Nakashima et al., 2009; Lee et al., 2012). The results obtained in the previous articles demonstrate the individuality of the United Quality Latent Index (UQLI, $I_{i}^{q}$ ) of adaptability of D. antarctica of plants from different sites in the Argentine Islands region, the maritime Antarctic. Also, calculation algorithms of the United Quality Latent Indices of adaptability (UQLI) of plants to natural conditions and United Temperature Influence Index (UTII) on the soil surface in the plant's habitats on the adaptability of plants were described along with their application (Miryuta et al., 2019a; 2019b). The results obtained in the previous articles demonstrate the individuality of the United Quality Latent Index (UQLI, $I_{i}^{q}$ ) of adaptability of $D$. antarctica of plants from the Galindez Island, Argentine Islands, the maritime Antarctic.

But there is another task, to follow how the longterm conditions in different regions have influenced their accumulated in nature adaptive capacity to preservation. This is more interesting because genetic studies of this species have shown a fairly low genetic heterogeneity (Andreev et al., 2010; Volkov et al., 2010;
Navrotska et al., 2017). The results of our previous studies of individual $D$. antarctica plants' adaptability of different origins are presented in (Miryuta et al., 2016; 2017; Parnikoza et al., 2017).

That's why in our current paper, we are going to describe in detail the algorithm for calculation of the UQLI $\left(I_{i}{ }^{i}\right)$ of adaptability for each plant from seeds collected in different regions of maritime Antarctic under the same germination conditions and cultivation in vitro (each plant was germinated from a single seed). The aim of this study is to elucidate the relationship between basic genetic characteristics of plants: genome size (2C nuclear DNA values), number of chromosomes, heterogeneity by ISSR (Inter Simple Sequence Repeats) and IRAP (Inter Retrotransposone Amplified Polymorphism) markers and some indices of adaptability for genotypes with different chromosome numbers.

According to Tchuraev's hypothesis (Tchuraev, 2006a), the genetic changes acquired through external conditions are saved in dynamic hereditary memory. Therefore, in this research, we tried to find out what $D$. antarctica's basic genetic characteristics characterize it under natural conditions that can be stored in the dynamic hereditary memory of each genotype. Dynamic hereditary memory is a way of saving information, which, unlike structural memory (when information is recorded in the spatial structure of biopolymers), is provided by circulating signals in a cyclic system of elements (Tchuraev, 2006a; 2006b). Hereinafter, the term 'genotype' will be understood as a genes and epigenes sets' characteristic for a plant obtained from a single seed and then cultivated in vitro.

Our selected adaptability indices were: leaf length, flavonoid content, and photosynthetic pigments content are related by auxin metabolism. In particular, flavonoids are such signaling molecules in auxin metabolism, which is responsible for plant growth. The growth rates of plants and their organs are controlled especially by auxin (indolyl-3-acetic acid - IAA), and flavonoids, coumarins and oxybenzoic acids are regulators of auxin metabolism. Quercetin, apigenin and kaempferol also interact with the NPA receptor in the plasma membrane of the plant cell and block the polar transport of auxin (Makarenko \& Levitsky, 2013). 
It is known from the literature that there is an interdependence between the production of phenolic compounds and morphometric parameters (Lambers et al., 2008). In particular, the length of the leaves is regulated by auxins, and the synthesis of auxins in the plant in response to ultraviolet light is regulated by flavonoids. In particular, luteolin is a synergist of auxin IAA (indolyl-3-acetic acid), and leaf growth is stimulated as a result of their co-production. Another flavonoid, apigenin, inhibits the synthesis of IAA (indolyl3 -acetic acid) because it is a cofactor of IAA oxidase and, accordingly, an antagonist of IAA (Makarenko \& Levitsky, 2013). Due to apigenin's action, the plant leaves become shorter (Grotewold, 2006). Because protective proteins can promote the synthesis of adaptive compounds like flavonoids and phenols (Van Loon, 2009; Shalygo et al., 2012), we studied such an index as the relative content of protective proteins in different fractions in the leaves (Miryuta et al., 2017).

It should be noted that we compare adaptive parameters indices data sets considered in the $\mathrm{m}$-formalism systems (the products of primary (proteins) and secondary (flavonoids) metabolism), which are system state variables (Tchuraev, 2006a) and the data sets in the L-formalism systems (the formation of a leaves system) that are system structure variables $(\mathrm{Zu}-$ bairova et al., 2012). For example, the state of each cell in the tissue can be characterized by the expression level of a set of particular genes. These indices will be each cell and tissue states variable. An example of a dynamic system is the description by means of these variables of tissue which functions in time and changes its state under the influence of external and/or internal reasons. Let us represent tissue as a system whose structure is determined by a cell-subsystems set between which there are certain connections (signal flows between neighboring cells). As a result of cell growth and division, the structure of the tissue changes, the environment of the cells changes, and accordingly, the signal flows between them change (Zubairova et al., 2012; 2014).

The way we have chosen to search for the basic genetic characteristics' influence on adaptive indices of different hierarchy levels is through creating correlation models visualized in probabilistic relationships graphs. Such models allow characterizing the heterogeneity of the studied genotypes, which should be taken into account in further studies.

\section{Materials and methods}

General characteristics of $D$. antarctica plants source data sets in vitro have been presented below. The ex-

Table 1. The populations' localization where the seeds have been collected for the experimental plants (genotypes) production, genome size $\left(\mathrm{Gs}_{\mathrm{i}}\right)$, and flavonoids content $\left(\mathrm{Fl}_{\mathrm{i}}\right)$ in Deschampsia antarctica plants grown in vitro (Parnikoza et al., 2017)

\begin{tabular}{|c|c|c|c|c|}
\hline $\mathrm{i}$ & Genotype & Localization, seed collection season & $\mathrm{Gs}_{\mathrm{i}}, \mathrm{pg}$ & $\mathrm{Fl}_{\mathrm{i}}, \mathrm{mg} / \mathrm{g}$ \\
\hline 1 & G/D4-1 & Galindez Island, $-65.248600^{\circ},-64.238217^{\circ}, 2012 / 13$ & $11.01 \pm 0.03$ & $2.92 \pm 0.70$ \\
\hline 2 & G/D12-2a & Galindez Island, $-65.247417^{\circ},-64.252600^{\circ}, 2006 / 07$ & $10.84 \pm 0.09$ & $2.8 \pm 0.61$ \\
\hline 3 & G/D12-1 & Galindez Island, $-65.247417^{\circ},-64.252600^{\circ}, 2013 / 14$ & $11.02 \pm 0.06$ & $3.86 \pm 0.70$ \\
\hline 4 & Y62 & $\begin{array}{l}\text { Great Yalour Island (our name for the biggest of Yalour Islands), } \\
-65.233983^{\circ},-64.162683^{\circ}, 2004 / 05\end{array}$ & $10.85 \pm 0.10$ & $1.62 \pm 0.40$ \\
\hline 5 & Y66 & Great Yalour Island, $-65.233983^{\circ},-64.162683^{\circ}, 2004 / 05$ & $16.74 \pm 0.07$ & $2.23 \pm 0.23$ \\
\hline 6 & Y67 & Great Yalour Island, $-65.233983^{\circ},-64.162683^{\circ}, 2004 / 05$ & $10.79 \pm 0.07$ & $2.64 \pm 0.53$ \\
\hline 7 & S22 & Skua Island, Finger Point, $-65.254933^{\circ},-64.274017^{\circ}, 2007 / 08$ & $10.94 \pm 0.04$ & $3.74 \pm 0.60$ \\
\hline 8 & R35 & Rasmussen Point, $-65.246983^{\circ},-64.085933^{\circ}, 2004 / 05$ & $10.77 \pm 0.02$ & $2.72 \pm 0.44$ \\
\hline 9 & W1 & Winter Island, $-65.247517^{\circ},-64.258033^{\circ}, 2013 / 14$ & $10.91 \pm 0.04$ & $2.23 \pm 0.28$ \\
\hline 10 & DAR12 & Darboux Island, $-65.395117^{\circ},-64.215083^{\circ}, 2006 / 07$ & $10.86 \pm 0.04$ & $1.22 \pm 0.15$ \\
\hline 11 & L59 & Lahille Island, $-65.553641^{\circ},-64.394930^{\circ}, 2009 / 10$ & $11.01 \pm 0.12$ & $4.67 \pm 0.30$ \\
\hline
\end{tabular}


perimental plants were grown in vitro as described in (Zahrychuk et al., 2011-2012).

We used the following data sets in our work:

$\mathrm{Gs}_{\mathrm{i}}$ were the genome size, where $i=1.2, \ldots \mathrm{n}$ (in this case $n=11$ ) and the values corresponding to the number of the studied genotypes G/D4-1, G/D122a, G/D12-1, Y62, Y66, Y67, S22, R35, W1, DAR12, L59 (Table 1) in this series of data and others (Parnikoza et al., 2017). Genome size (2C value of nuclear DNA) for each genotype of D. antarctica was calculated based on the method of flow cytometry (Bai et al., 2012);

$\mathrm{Ph}_{\mathrm{i}}$ were morphometric parameter leaf length where $i=1.2, \ldots \mathrm{n}$ (in this case $\mathrm{n}=11$ ) and the values corresponding to the number of the studied genotypes (Table 1). $\mathrm{Ph}_{\mathrm{i}}$ sets data used in our work have been obtained by methods described in (Parnikoza et al., 2015);

$\mathrm{Fl}_{\mathrm{i}}$ were flavonoid content values in leaves (Table 1) (Poronnik et al., 2017; Ermakov, 1987). The leaf length and flavonoid content in leaves that are connected with auxin metabolism we will call auxin metabolism-related indices (Makarenko \& Levitsky, 2013; Zubairova et al., 2012; 2014);

$\operatorname{Pr}_{\mathrm{ik}}$ were the relative content values of protective and basic $(\mathrm{RuBis} \mathrm{CO})$ proteins in the leaves, where $k=$ $=1,2, \ldots 6$ (the number of the corresponding protein by size in $\mathrm{kDa}$ to: $66-67$ is large hs-protein-chaperone $\left(\operatorname{Pr}_{i 1}\right), 45$ is $\mathrm{RuBisCO}$ (small subunit)) $\left(\operatorname{Pr}_{\mathrm{i} 2}\right), 36$ is one of the antifreeze proteins (in Secale cereale L., 1753) $\left(\operatorname{Pr}_{\mathrm{i} 3}\right) ; 24$ is one of the chaperones Triticum aestivum, L., $1753\left(\operatorname{Pr}_{\mathrm{i} 4}\right)$; 20-22 is one of the antifreeze proteins of $D$. antarctica $\left(\operatorname{Pr}_{\mathrm{i} 5}\right) ; 14$ is small hs-protein, dehydrin $\left(\operatorname{Pr}_{i 6}\right)$. $\operatorname{Pr}_{\mathrm{ik}}$ data sets were obtained by methods described in (Miryuta et al., 2016; 2017; Scion Image http://scion-image.software.informer.com/ 4.0/);

$\Delta \mathrm{Glg}_{\mathrm{i}}$ were data on genetic distances. Data were taken from the article (Navrotska et al., 2017) and obtained according to Jacquard method between plants of different $D$. antarctica genotypes, calculated by the results of ISSR and IRAP analysis (Navrotska et al., 2017), $i=4,5, \ldots \mathrm{n}(\mathrm{n}=11)$;

$\mathrm{Pg}_{\mathrm{ij}}$ were the content of photosynthetic pigments, where $j=1,2,3$ where pigment number corresponds to $j=1$ is chlorophyll A (ChlA), $j=2$ is chlorophyll $\mathrm{B}$ (ChlB), $j=3$ is carotenoids (Car) (Poronnik et al., 2019).
The data sets for $\mathrm{Gs}_{\mathrm{i}}, \mathrm{Fl}_{\mathrm{i}}$, and $\Delta \mathrm{Glg}_{\mathrm{i}}$ are shown in Tables 1 and 2 correspondingly, for $\mathrm{Ph}_{\mathrm{i}}, \mathrm{Pr}_{\mathrm{ik}}, \mathrm{Pg}_{\mathrm{ij}}$, are shown in Figures 1-3.

Variability of plants based on molecular markers was studied by PCR analysis with ISSR and IRAP primers. In total, 63 amplicons were obtained for the investigated samples, among which 22 (34.2\%) were polymorphic (Table 2) (Navrotska et al., 2017).

\section{Results and discussion}

This work is a continuation of the works series devoted to algorithms described for calculating in detail the United Quality Latent Index (UQLI, I ${ }^{\mathrm{q}}$ ) of plant adaptability to the environment on Argentine Islands, maritime Antarctic (Miryuta et al., 2019a; 2019b). In our study, all environmental factors were anonymous (latent), not measured. At the same time, we used as a basis the thesis that we considered the existing organism as adapted to these factors. Thus, we investigated the finish of such adaptation results based on signs that were available for measurement. Of course, this approach is simplified and does not consider a detailed study of all mechanisms. Still, it is used in classical applied statistics (Ayvazyan et al., 1989; Bauman \& Moskalenko, 2008), so the name United Quality Latent Indices of the plant adaptability actually means a complex adaptability index based on the measured parameters (Miryuta et al., 2019a). In the second work of this series, we investigated the temperature influence on each of the measured indices of plants, calculating the United Temperature on the soil surface Influence Index (UTII) on the population plants and calculating the contribution of UTII to UQLI (Miryuta et al., $2019 b)$. The algorithms calculation of the UQLI of plants adaptability to natural conditions and UTII on the soil surface in places of population growth on the plants' adaptability and contribution UTII to UQLI were described in these works.

Turning to the study of $D$. antarctica plants in the laboratory, which is associated with the difficulty of plant material delivery from the Argentine Islands, we rely on the following hypothesis. According to Tchuraev's hypothesis, the existence of epigenes and functional hereditary memory makes it possible to imple- 


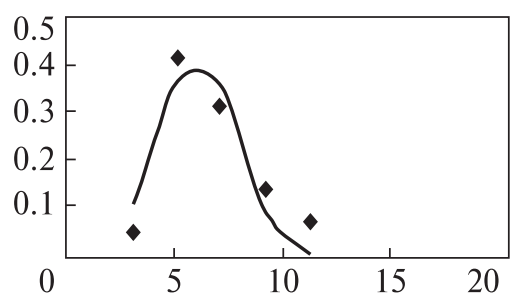

(a)

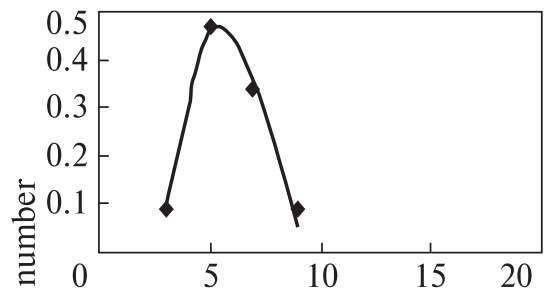

(d)

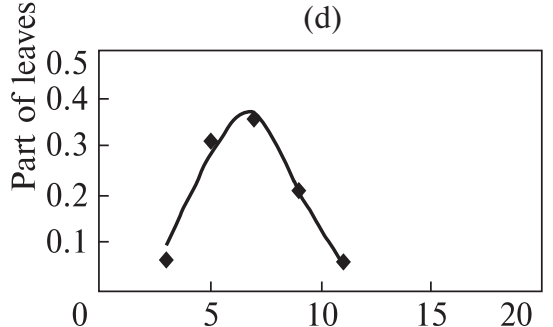

(g)

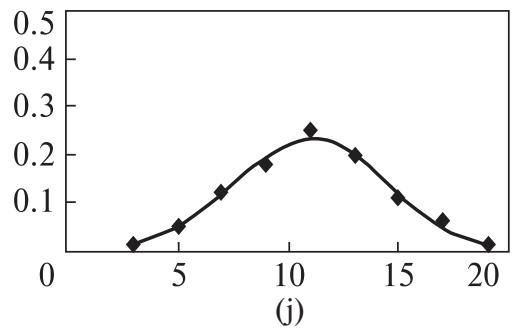

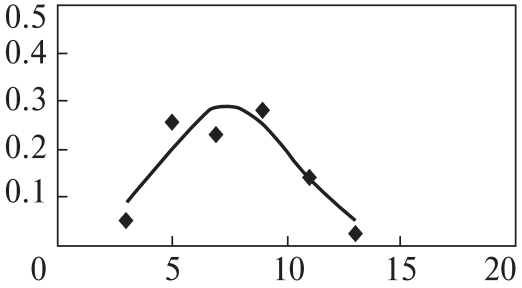

(b)

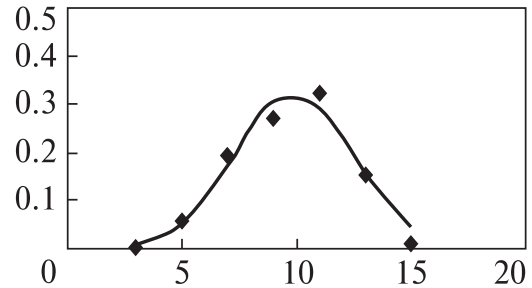

(e)

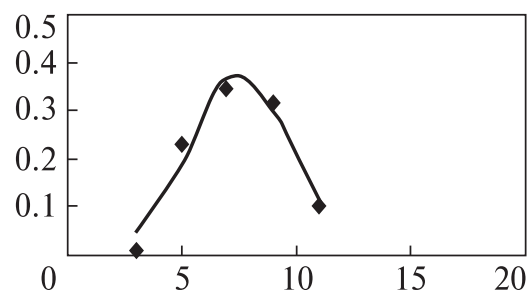

(h)

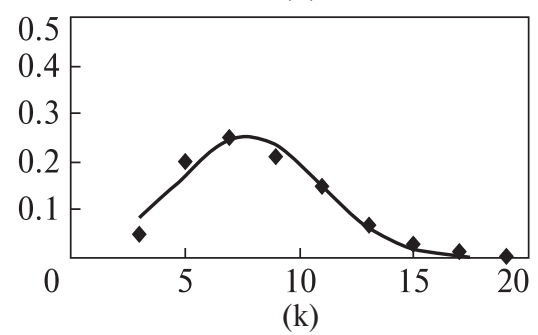

Leaf length, cm

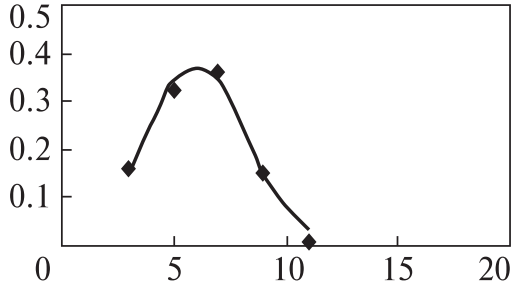

(c)

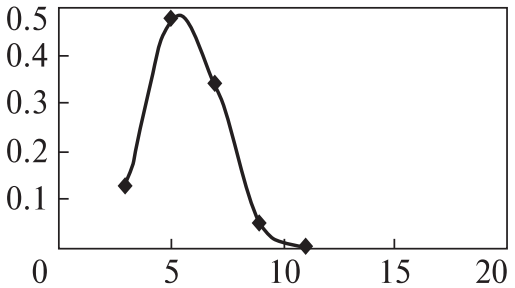

(f)

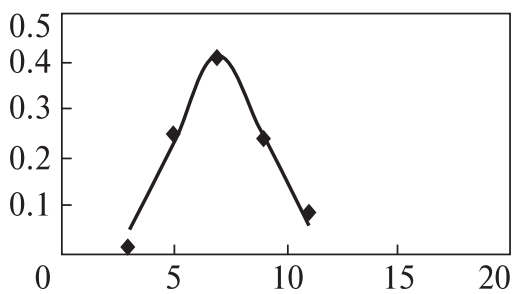

(i)

Figure 1. Density functions (Gaussian models) based on leaf length (horizontally, $\mathrm{cm}$ ) of Deschampsia antarctica plants of different genotypes cultivated in vitro: ((a) - G/D4-1, (b) - G/D12-2a, (c) - G/D12-1) plants from Galindez Island, ((d) Y62, (e) - Y66, (f) - Y67) plants from Great Yalour Island, ((g) - S22, (h) - R35, (i) - W1, (j) - DAR12, (k) - L59) plants from Skua Island, Rasmussen Point, Winter, Darboux, and Lahille Islands, respectively. Vertically: part of leaves number (by Navrotska et al., 2017)

ment a non-Darwinian evolutionary strategy when relatively unsuccessful moves in the hereditary memory of individuals are not 'forgotten', and the corresponding subroutines remain off in functional hereditary memory without reflection in ontogenesis (Tchuraev, 2006a).

The dependence of plants on both growth microenvironment conditions and the weather conditions of the study season becomes irrelevant in the laboratory, but it is assumed that information is saved in the dynamic hereditary memory of seeds both about the influence of growth microconditions and weather conditions of the season of seed ripening. In this study, we aimed to test the extent to which the origin of seeds affects the uniqueness of the realization of the hereditary information of genotypes derived from it and cultured in the laboratory.

This work deals with describing in detail the algorithm for calculating the UQLI $\left(I^{q}\right)$ of plants adapt- 


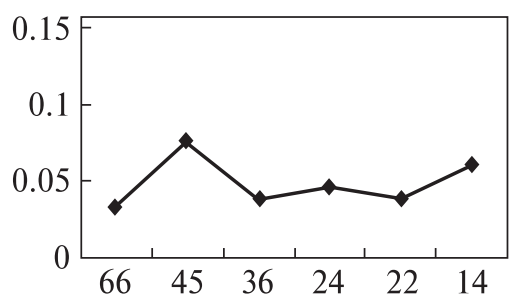

(a)

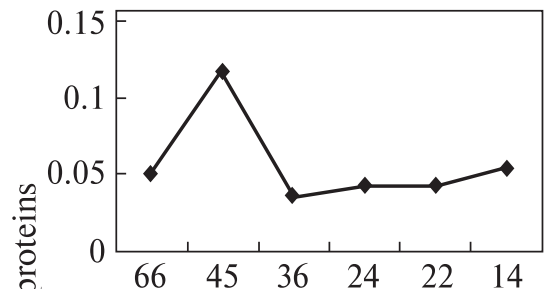

(d)

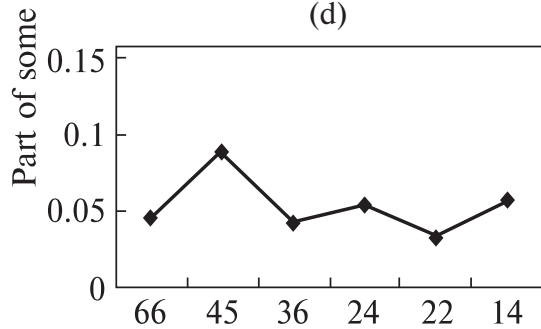

(g)

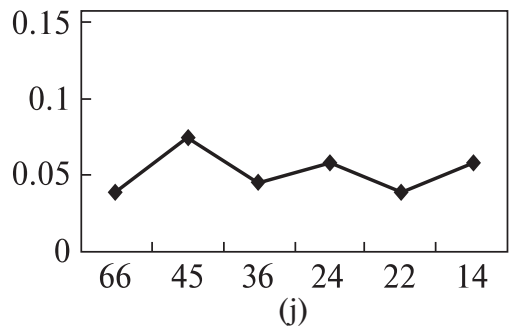

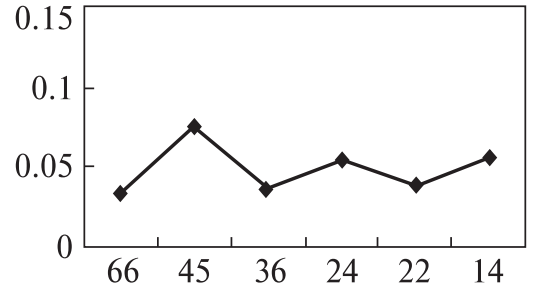

(b)

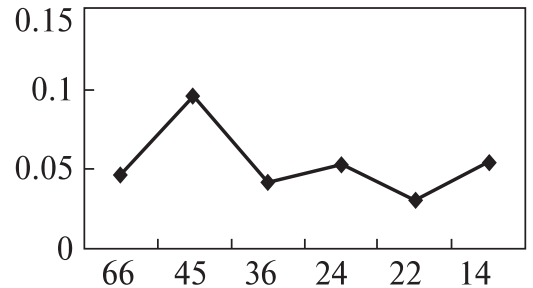

(e)

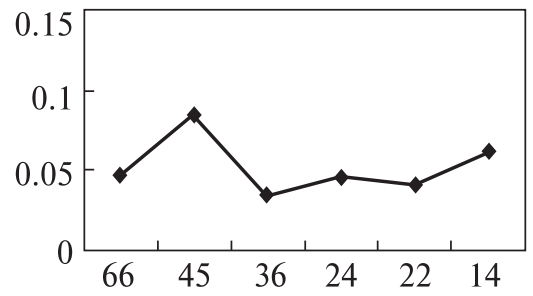

(h)

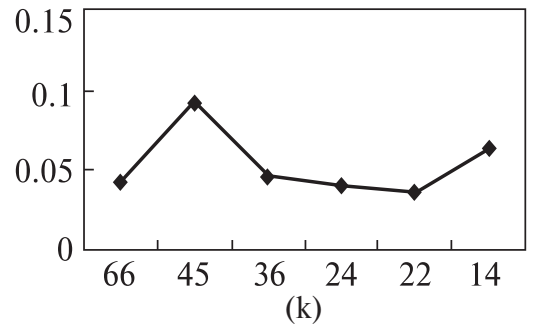

Approximate protein size, $\mathrm{kDa}$

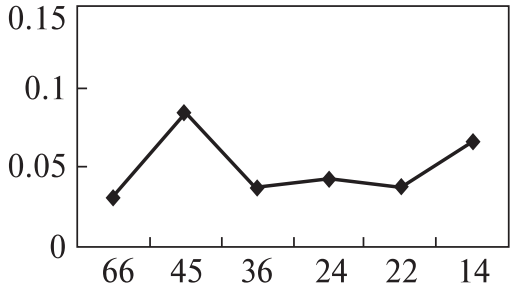

(c)

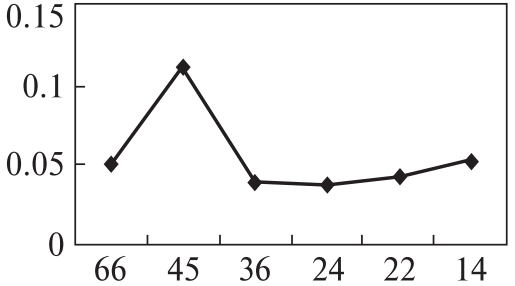

(f)

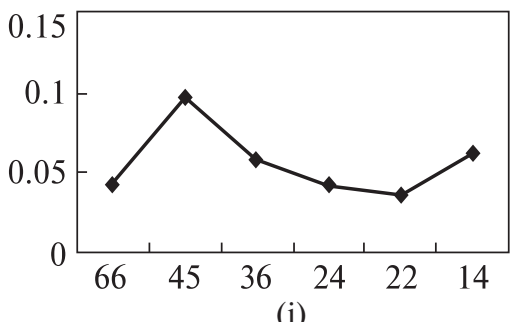

(i)

Figure 2. The values of the different protein fractions parts (proportions) in the leaves of Deschampsia antarctica plants under cultivation in vitro. The studied genotypes were: (a) - G/D4-1, (b) - G/D12-2a, (c) - G/D12-1, (d) - Y62, (e) - Y66, (f) Y67, (g) - S22, (h) - R35, (i) - W1, (j) - DAR12, (k) - L59. The sizes of proteins in kDa were presented: 66-67 is a large hs-protein-chaperone; 45 - RuBisCO (small subunit); 36 - one of the antifreeze proteins (in Secale cereale L., 1753 ); 24 one of the chaperones of Triticum aestivum L. 1753; 20-22 - one of the antifreeze proteins of Deschampsia antarctica; 14 - small hs-protein, dehydrin

ability from seeds collected in different regions of the maritime Antarctic under the same germination conditions and cultivation in vitro. The aim of this study is to find the relationship between the genome size and genetic distances integral indices, and some adaptability indices.

D. antarctica plants obtained from seeds from different populations can store specific information in the dynamic hereditary memory embedded under the process of adaptation of plants to unique natural conditions under in vitro cultivation (Tchuraev, 2006a). In such case, most likely, the UQLI and its components will be highly individual. Dynamic hereditary memory is a way to store information which, unlike structural memory (when information is written in the linear and spatial structure of biopolymers), is provided by 


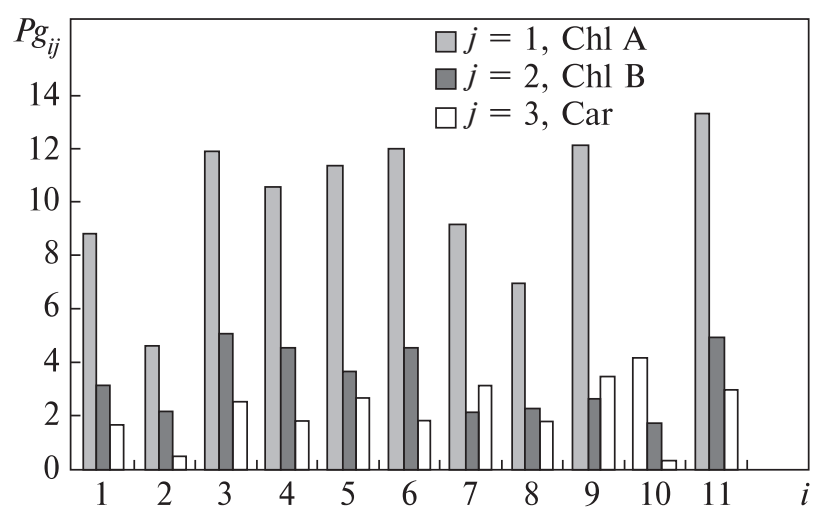

Figure 3. Average values of the photosynthetic pigments content $\left(\mathrm{Pg}_{\mathrm{ij}}\right)$ in the leaves of Deschampsia antarctica plants under cultivation in vitro, where $j=1,2,3$ pigment number corresponds to chlorophyll A (ChlA), chlorophyll B (ChlB), carotenoids (Car) (Poronnik et al., 2019). The studied genotypes by $i$ were: $1-\mathrm{G} / \mathrm{D} 4-1,2-\mathrm{G} / \mathrm{D} 12-2 \mathrm{a}, 3-\mathrm{G} / \mathrm{D} 12-1,4-$ Y62, 5 - Y66, $6-\mathrm{Y} 67,7-\mathrm{S} 22,8-\mathrm{R} 35,9-\mathrm{W} 1,10-$ DAR12, 11 - L59

circulating signals in a cyclic system of elements. An example would be cyclic gene systems that have at least two functional states and can maintain each state both throughout the cell's existence and during successive cell divisions. Due to the difference between structural and dynamic heredity in addition to the term 'gene' (meaning a unit of transcription and transmitted to offspring by the primary DNA sequence), the term 'epigene' was introduced for part of hereditary information that is stored and passed on to offspring out of DNA molecules structure. An epi- genome is a hereditary unit with at least two functioning modes of its subordinate genes and can store each of the modes in a successive generations series (Tchuraev, 2006a).

Hereinafter, the term 'genotype' will be understood as genes and epigenes sets which characterize a plant obtained from a single seed followed by its cloning in vitro. Applying this conception in the UQLI calculation, it is particularly interesting to analyze the components of this integrated index, which shows the probability of positive (difference of indices in phase) or negative (difference of indices in antiphase) relationship between the studied indices with different hierarchical levels.

In further studies, it will be possible to influence different genotypes of plants by varying biotic and abiotic factors and to investigate how UQLI components change.

The advantage of $D$. antarctica plants studies under cultivation in vitro is the ability to expand the range of studied characteristics. This circumstance makes it possible to investigate a larger number of probabilistic relationships between different parameters (UQLI components). However, the same circumstance complicates the graphical representation of the studied material. Therefore, we present a graphical version of the algorithm for determining UQLI for the simplest case, which consists of three measured characteristics: genome size $\left(\mathrm{Gs}_{\mathrm{i}}\right)$, leaf length (in the form of leaf length distributions) $\left(\mathrm{Ph}_{\mathrm{i}}\right)$, and

Table 2. Genetic distances $\left(\Delta \mathrm{Glg}_{\mathrm{i}}\right)$ by Jacquard between plants of different Deschampsia antarctica genotypes (Navrotska et al., 2017)

\begin{tabular}{|l|c|c|c|c|c|c|c|c|}
\hline \multicolumn{1}{|c|}{$\begin{array}{c}\text { Genotype } \\
\text { name }\end{array}$} & Y62 & Y66 & Y67 & S22 & R35 & W1 & DAR12 & L59 \\
\hline Y62 & 0 & 0.0476 & 0.0484 & 0.0484 & 0.0476 & 0.1290 & 0.0806 & 0.0645 \\
Y66 & 0.0476 & 0 & 0.0641 & 0.0645 & 0.0323 & 0.1452 & 0.0968 & 0.0806 \\
Y67 & 0.0484 & 0.0641 & 0 & 0.0968 & 0.0645 & 0.1475 & 0.0983 & 0.0500 \\
S22 & 0.0484 & 0.0645 & 0.0968 & 0 & 0.0952 & 0.0847 & 0.0984 & 0.1129 \\
R35 & 0.0476 & 0.0323 & 0.0645 & 0.0952 & 0 & 0.1746 & 0.1270 & 0.0806 \\
W1 & 0.1290 & 0.1452 & 0.1475 & 0.0847 & 0.1746 & 0 & 0.1803 & 0.1639 \\
DAR12 & 0.0806 & 0.0968 & 0.0983 & 0.0984 & 0.1270 & 0.1803 & 0 & 0.1148 \\
L59 & 0.0645 & 0.0806 & 0.0500 & 0.1129 & 0.0806 & 0.1639 & 0.1148 & 0 \\
\hline
\end{tabular}


the total flavonoid content in the leaves $\left(\mathrm{Fl}_{\mathrm{i}}\right)$ (Fig. 4). In the example below, the relative content of protective and basic proteins in the leaves $\left(\operatorname{Pr}_{\mathrm{ik}}\right)$, genetic heterogeneity in the form of genetic distances between eight genotypes $\left(\Delta \mathrm{Glg}_{\mathrm{i}}\right)$, photosynthetic pigments $\left(\mathrm{Pg}_{\mathrm{ij}}\right)$ were added in turn.

An algorithm for determining $D$. antarctica plants genotypes' UQLI under cultivation in vitro based on the experimental material published in the series of articles (Miryuta et al., 2016; 2017) was developed. An example of the algorithm for three indices is presented in Figure 4. A group of 11 genotypes was selected for the research; the list and coordinates of sites of origin are presented in Table 1.

We would consider the graph shown in Figure 4 step by step.

1. Source data sets. Examples of data sets are partially presented in Tables 1 and $2\left(\mathrm{Gs}_{\mathrm{i}}, \mathrm{Fl}_{\mathrm{i}}, \Delta \mathrm{Glg}_{\mathrm{i}}\right)$ and Figures 1-3 $\left(\mathrm{Ph}_{\mathrm{ij}}, \mathrm{Pr}_{\mathrm{ik}}\right.$, and $\left.\mathrm{Pg}_{\mathrm{ij}}\right)$.

2. Spatial pairwise comparison of the source data sets (by $i$ ). Pairwise spatial differences in the modulus for $\mathrm{Gs}_{\mathrm{i}}, \mathrm{Fl}_{\mathrm{i}}, \mathrm{Pr}_{\mathrm{ik}}$, and $\mathrm{Pg}_{\mathrm{ij}}$ data sets have been found. The test value for pairwise comparison of distributions for the data set $\mathrm{Ph}_{\mathrm{i}}$ has been found by the Mood median test. This nonparametric test was a variation of the $\chi^{2}$ test, which allows estimating intra-group differences for two genotypes without assessing the normal distribution of genotype indices (Pollard, 1982; Corder \& Foreman, 2014). The table included the values of criterion statistics (which is proportional to the distance between the medians), which exceeded the value of the table value 3.84 by $\chi^{2}$ test (Pollard, 1982). The resulting sets of pairwise spatial comparisons were denoted by $\Delta \mathrm{Glg}_{\mathrm{i}}, \Delta \mathrm{Gs}_{\mathrm{i}}, \Delta \mathrm{Fl}_{\mathrm{i}}, \Delta \mathrm{Pr}_{\mathrm{ik}}$, and $\Delta \mathrm{Pg}_{\mathrm{ij}}$. Examples of such comparisons are presented in Tables 2-4.

3. Extreme grouping of points in pairwise spatial differences indices sets pairs. To reduce the dimensions of the studied space values described by indices, the number of which is gradually increasing, the method of extreme grouping of points in pairs indices sets of characteristics pairs differences for cultivated in vitro plant genotypes have been used (Ayvazyan et al., 1989; Bauman \& Moskalenko, 2008).

In contrast to plants in populations that exist under natural conditions, a set of genotypes from differ-
Source data sets

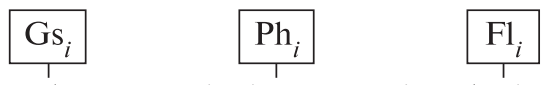

Source data sets pairwise comparison by index $i$, where $i$ is genotype number

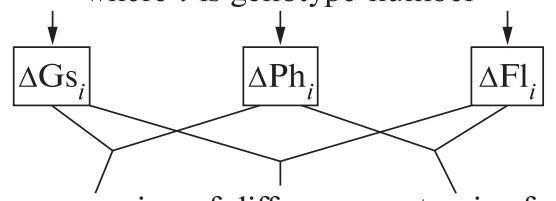

Extreme grouping of differences set pair of source data by index $i$ in studied genotypes

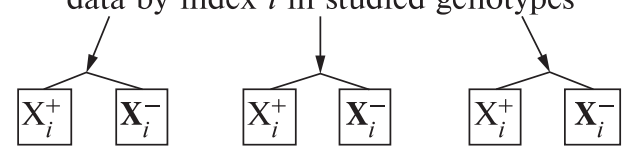

Determination of each $i$-th genotype probability of matching into a positive or negative group

$$
\begin{array}{|l|l|}
X_{i l(l=1)} & X_{i l(l=2)} \\
\hline
\end{array}
$$

Determination of United Quality Indices for each pair of differences sets for the $i$-th genotype

$$
\begin{array}{|l|l|}
\hline I_{i l(l=1)}^{q} & I_{i l(l=2)}^{q} \\
\hline
\end{array}
$$

Determination of United Quality Indicex for the $i$-th genotype

$$
I_{i}^{q}=\frac{2 !(l-2) !}{l !} \sum_{l} I_{i l}^{q}
$$

Figure 4. The simplest example of an algorithm for calculating UQLI for plant genotypes from seeds of different populations under cultivation in vitro

ent natural habitats cultivated in vitro have adaptability indices that reflect the properties acquired by plants and recorded in dynamic hereditary memory as one of the options that can be implemented. Adaptability indices of such plants under growing conditions in vitro were also described indirectly but reflect one from the many options that were implemented under the created conditions in contrast to plants under natural conditions when they were influenced by a range of changing environment indices. That's why the results were not easy to interpret. First, sets of pairwise comparisons of all studied genotypes were grouped by three sets pairs of adaptability indices: $\Delta \mathrm{Ph}_{\mathrm{i}}-\left|\Delta \mathrm{Gs}_{\mathrm{i}}\right|,\left|\Delta \mathrm{F} l_{\mathrm{i}}\right|-\left|\Delta \mathrm{Gs}_{\mathrm{i}}\right|, \Delta \mathrm{Ph}_{\mathrm{i}}-\left|\Delta \mathrm{F} \mathrm{l}_{\mathrm{i}}\right|$. Second, when we considered four adaptability indices sets the grouping was performed by six adaptability indices pairs: $\left|\Delta \mathrm{Gs}_{\mathrm{i}}\right|$ versus $\left|\Delta \mathrm{Ph}_{\mathrm{i}}\right|,\left|\Delta \mathrm{Gs}_{\mathrm{i}}\right|$ versus $\left|\Delta \mathrm{Pr}_{\mathrm{ik}}\right|,\left|\Delta \mathrm{Gs}_{\mathrm{i}}\right|$ versus $\left|\Delta \mathrm{Fl}_{\mathrm{i}}\right|,\left|\Delta \mathrm{Ph}_{\mathrm{i}}\right|$ versus $\left|\Delta \mathrm{Pr}_{\mathrm{ik}}\right|,\left|\Delta \mathrm{Fl}_{\mathrm{i}}\right|$ versus $\mid \Delta \mathrm{Pr}_{\mathrm{ik}}$, $\left|\Delta \mathrm{Fl}_{\mathrm{i}}\right|$ versus $\left|\Delta \mathrm{Ph}_{\mathrm{i}}\right|$ or $\left|\Delta \mathrm{Gs}_{\mathrm{i}}\right|$ versus $\left|\Delta \mathrm{Ph}_{\mathrm{i}}\right|,\left|\Delta \mathrm{Gs}_{\mathrm{i}}\right|-$ $\left|\Delta \mathrm{Glg}_{\mathrm{i}}\right|,\left|\Delta \mathrm{Gs}_{\mathrm{i}}\right|-\left|\Delta \mathrm{Fl}_{\mathrm{i}}\right|,\left|\Delta \mathrm{Ph}_{\mathrm{i}}\right|-\left|\Delta \mathrm{Glg}_{\mathrm{i}}\right|,\left|\Delta \mathrm{Fl}_{\mathrm{i}}\right|-\left|\Delta \mathrm{Glg}_{\mathrm{i}}\right|$, 
Table 3. The differences (by value $i$ ) of genome size $\left(\Delta \mathrm{Gs}_{\mathrm{i}}\right)$ and relative content of protective and main proteins in leaves $\left(\Delta \mathrm{Pr}_{\mathrm{ik}}\right)$

\begin{tabular}{|c|c|c|c|c|c|c|c|}
\hline \multirow{2}{*}{$\Delta i$} & \multirow{2}{*}{$\left|\Delta\left(\mathrm{Gs}_{\mathrm{i}}\right)\right|$} & \multicolumn{6}{|c|}{$\left|\Delta \mathrm{Pr}_{\mathrm{ik}}\right|, \mathrm{kDa}$} \\
\hline & & $66-67(k=1)$ & $45(k=2)$ & $36(k=3)$ & $24(k=4)$ & $22(k=5)$ & $14(k=6)$ \\
\hline G/D4-1-G/D12-2a & 0.170 & 0.0 & 0.0 & 0.002 & 0.008 & 0.008 & 0.004 \\
\hline G/D4-1-G/D12-1 & 0.010 & 0.002 & 0.008 & 0.001 & 0.007 & 0.003 & 0.003 \\
\hline G/D4-1-Y62 & 0.160 & 0.017 & 0.041 & 0.002 & 0.004 & 0.002 & 0.005 \\
\hline G/D4-1-Y66 & 5.730 & 0.013 & 0.020 & 0.003 & 0.005 & 0.001 & 0.002 \\
\hline G/D4-1-Y67 & 0.220 & 0.018 & 0.036 & 0.000 & 0.001 & 0.006 & 0.011 \\
\hline G/D4-1-S22 & 0.070 & 0.012 & 0.013 & 0.004 & 0.016 & 0.012 & 0.002 \\
\hline G/D4-1-R35 & 0.240 & 0.014 & 0.009 & 0.003 & 0.001 & 0.003 & 0.002 \\
\hline G/D4-1-W1 & 0.100 & 0.009 & 0.021 & 0.020 & 0.010 & 0.003 & 0.002 \\
\hline G/D4-1-DAR12 & 0.150 & 0.005 & 0.001 & 0.007 & 0.009 & 0.003 & 0.005 \\
\hline G/D4-1-L59 & 0.000 & 0.010 & 0.017 & 0.008 & 0.009 & 0.000 & 0.003 \\
\hline G/D12-2A-G/D12-1 & 0.180 & 0.002 & 0.008 & 0.001 & 0.012 & 0.002 & 0.007 \\
\hline G/D12-2A-Y62 & 0.010 & 0.017 & 0.041 & 0.000 & 0.015 & 0.002 & 0.008 \\
\hline G/D12-2A-Y66 & 5.900 & 0.013 & 0.020 & 0.005 & 0.012 & 0.001 & 0.010 \\
\hline G/D12-2A-Y67 & 0.050 & 0.018 & 0.036 & 0.002 & 0.008 & 0.001 & 0.005 \\
\hline G/D12-2A-S22 & 0.100 & 0.012 & 0.013 & 0.006 & 0.013 & 0.005 & 0.001 \\
\hline G/D12-2A-R35 & 0.070 & 0.014 & 0.009 & 0.001 & 0.018 & 0.004 & 0.003 \\
\hline G/D12-2A-W1 & 0.070 & 0.009 & 0.021 & 0.022 & 0.009 & 0.001 & 0.008 \\
\hline DAR12-G/D12-2a & 0.020 & 0.005 & 0.001 & 0.009 & 0.006 & 0.008 & 0.004 \\
\hline G/D12-2A-L59 & 0.170 & 0.010 & 0.017 & 0.010 & 0.007 & 0.011 & 0.006 \\
\hline G/D12-1-Y62 & 0.170 & 0.019 & 0.033 & 0.001 & 0.001 & 0.007 & 0.008 \\
\hline G/D12-1-Y66 & 5.720 & 0.015 & 0.012 & 0.004 & 0.010 & 0.006 & 0.008 \\
\hline G/D12-1-Y67 & 0.230 & 0.020 & 0.028 & 0.001 & 0.006 & 0.006 & 0.010 \\
\hline G/D12-1-S22 & 0.080 & 0.014 & 0.005 & 0.005 & 0.011 & 0.003 & 0.006 \\
\hline G/D12-1-R35 & 0.250 & 0.016 & 0.001 & 0.002 & 0.003 & 0.005 & 0.002 \\
\hline G/D12-1-W1 & 0.110 & 0.011 & 0.013 & 0.021 & 0.003 & 0.000 & 0.001 \\
\hline G/D12-1-DAR12 & 0.160 & 0.007 & 0.009 & 0.008 & 0.016 & 0.002 & 0.004 \\
\hline G/D12-1-L59 & 0.010 & 0.012 & 0.009 & 0.009 & 0.004 & 0.003 & 0.002 \\
\hline Y62-Y66 & 5.890 & 0.004 & 0.021 & 0.005 & 0.013 & 0.006 & 0.009 \\
\hline Y62-Y67 & 0.060 & 0.001 & 0.005 & 0.002 & 0.003 & 0.006 & 0.011 \\
\hline Y62-S22 & 0.090 & 0.005 & 0.028 & 0.006 & 0.014 & 0.003 & 0.007 \\
\hline Y62-R35 & 0.080 & 0.003 & 0.032 & 0.001 & 0.006 & 0.005 & 0.003 \\
\hline Y62-W1 & 0.060 & 0.008 & 0.020 & 0.022 & 0.000 & 0.001 & 0.003 \\
\hline Y62-DAR12 & 0.010 & 0.012 & 0.042 & 0.009 & 0.019 & 0.002 & 0.005 \\
\hline Y62-L59 & 0.160 & 0.007 & 0.024 & 0.010 & 0.003 & 0.001 & 0.002 \\
\hline Y66-Y67 & 5.950 & 0.005 & 0.016 & 0.003 & 0.006 & 0.009 & 0.006 \\
\hline Y66-S22 & 5.800 & 0.001 & 0.007 & 0.001 & 0.011 & 0.004 & 0.009 \\
\hline Y66-R35 & 5.970 & 0.001 & 0.011 & 0.006 & 0.003 & 0.004 & 0.005 \\
\hline
\end{tabular}


End of Table 3

\begin{tabular}{|c|c|c|c|c|c|c|c|}
\hline \multirow{2}{*}{$\Delta i$} & \multirow{2}{*}{$\left|\Delta\left(\mathrm{Gs}_{\mathrm{i}}\right)\right|$} & \multicolumn{6}{|c|}{$\left|\Delta \mathrm{Pr}_{\mathrm{ik}}\right|, \mathrm{kDa}$} \\
\hline & & $66-67(k=1)$ & $45(k=2)$ & $36(k=3)$ & $24(k=4)$ & $22(k=5)$ & $14(k=6)$ \\
\hline Y66-W1 & 5.830 & 0.004 & 0.001 & 0.017 & 0.010 & 0.003 & 0.008 \\
\hline Y66-DAR12 & 5.880 & 0.008 & 0.021 & 0.004 & 0.016 & 0.001 & 0.007 \\
\hline Y66-L59 & 5.730 & 0.003 & 0.003 & 0.005 & 0.006 & 0.005 & 0.013 \\
\hline Y67-S22 & 0.150 & 0.006 & 0.023 & 0.004 & 0.007 & 0.006 & 0.004 \\
\hline Y67-R35 & 0.020 & 0.004 & 0.027 & 0.003 & 0.001 & 0.002 & 0.000 \\
\hline Y67-W1 & 0.120 & 0.009 & 0.015 & 0.020 & 0.002 & 0.007 & 0.009 \\
\hline Y67-DAR12 & 0.070 & 0.013 & 0.037 & 0.007 & 0.012 & 0.001 & 0.002 \\
\hline Y67-L59 & 0.220 & 0.008 & 0.019 & 0.008 & 0.005 & 0.004 & 0.006 \\
\hline S22-R35 & 0.170 & 0.002 & 0.004 & 0.007 & 0.004 & 0.002 & 0.006 \\
\hline S22-W1 & 0.030 & 0.003 & 0.008 & 0.016 & 0.010 & 0.007 & 0.011 \\
\hline S22-DAR12 & 0.080 & 0.007 & 0.014 & 0.003 & 0.017 & 0.005 & 0.004 \\
\hline S22-L59 & 0.070 & 0.002 & 0.004 & 0.004 & 0.011 & 0.013 & 0.000 \\
\hline R35-W1 & 0.140 & 0.005 & 0.012 & 0.023 & 0.012 & 0.010 & 0.002 \\
\hline R35-DAR12 & 0.090 & 0.009 & 0.010 & 0.010 & 0.022 & 0.004 & 0.006 \\
\hline R35-L59 & 0.240 & 0.004 & 0.008 & 0.011 & 0.017 & 0.009 & 0.004 \\
\hline W1-DAR12 & 0.050 & 0.004 & 0.022 & 0.013 & 0.002 & 0.008 & 0.001 \\
\hline W1-L59 & 0.100 & 0.001 & 0.004 & 0.012 & 0.003 & 0.005 & 0.002 \\
\hline DAR12-L59 & 0.150 & 0.005 & 0.018 & 0.001 & 0.001 & 0.005 & 0.001 \\
\hline
\end{tabular}

Table 4. Differences (by value $i$ ) of genome size $\left(\Delta \mathrm{Gs}_{\mathrm{i}}\right)$, leaf length $\left(\Delta \mathrm{Ph}_{\mathrm{i}}\right)$, flavonoid content in leaves $\left(\Delta \mathrm{Fl}_{\mathrm{i}}\right)$, photosynthetic pigments content $\left(\Delta \mathrm{Pg}_{\mathrm{ij}}\right)$, and genetic distances according to Jacquard ( $\left.\Delta \mathrm{Glg}_{\mathrm{i}}\right)$ (Navrotska et al., 2017) for Deschampsia antarctica genotypes

\begin{tabular}{|l|c|c|c|c|c|c|c|}
\hline \multicolumn{1}{|c|}{$\Delta i$} & $\left|\Delta \mathrm{Gs}_{\mathrm{i}}\right|$ & $\Delta \mathrm{Ph}_{\mathrm{i}}$ & $\left|\Delta \mathrm{Fl}_{\mathrm{i}}\right|$ & $\Delta \mathrm{Glg}_{\mathrm{i}}$ & $\left|\Delta \mathrm{Pg}_{\mathrm{ij}}\right|(j=1)$ & $\left|\Delta \mathrm{Pg}_{\mathrm{i}}\right|(j=2)$ & $\left|\Delta \mathrm{Pg}_{\mathrm{ij}}\right|(j=3)$ \\
\hline G/D4-1-G/D12-2a & 0.17 & 21.68 & 0.12 & - & 4.16 & 0.98 & 1.12 \\
G/D4-1-G/D12-1 & 0.01 & 0.00 & 0.94 & - & 3.05 & 1.93 & 0.83 \\
G/D4-1-Y62 & 0.16 & 16.20 & 0.13 & - & 1.74 & 1.42 & 0.14 \\
G/D4-1-Y66 & 5.73 & 166.74 & 0.69 & - & 2.56 & 0.47 & 1.03 \\
G/D4-1-Y67 & 0.22 & 16.70 & 0.28 & - & 3.20 & 1.38 & 0.18 \\
G/D4-1-S22 & 0.07 & 0.00 & 0.82 & - & 0.32 & 1.02 & 1.48 \\
G/D4-1-R35 & 0.24 & 90.6 & 0.20 & - & 1.89 & 0.88 & 0.19 \\
G/D4-1-W1 & 0.10 & 10.58 & 0.69 & - & 3.31 & 0.54 & 1.80 \\
G/D4-1-DAR12 & 0.15 & 253.49 & 1.70 & - & 4.66 & 1.43 & 1.40 \\
G/D4-1-L59 & 0.00 & 66.35 & 1.75 & - & 4.55 & 1.79 & 1.31 \\
G/D12-2a-G/D12-1 & 0.18 & 29.04 & 1.06 & - & 7.21 & 2.91 & 1.95 \\
G/D12-2a-Y62 & 0.01 & 15.47 & 1.18 & - & 5.90 & 2.40 & 1.26 \\
G/D12-2a-Y66 & 5.90 & 22.77 & 0.57 & - & 6.72 & 1.45 & 2.15 \\
G/D12-2a-Y67 & 0.05 & 20.79 & 0.16 & - & 7.36 & 2.36 & 1.30
\end{tabular}


End of Table 4

\begin{tabular}{|c|c|c|c|c|c|c|c|}
\hline$\Delta i$ & $\left|\Delta \mathrm{Gs}_{\mathrm{i}}\right|$ & $\Delta \mathrm{Ph}_{\mathrm{i}}$ & $\left|\Delta \mathrm{Fl}_{\mathrm{i}}\right|$ & $\Delta \mathrm{Glg}_{\mathrm{i}}$ & $\left|\Delta \mathrm{Pg}_{\mathrm{ij}}\right|(j=1)$ & $\left|\Delta \mathrm{Pg}_{\mathrm{in}}\right|(j=2)$ & $\left|\Delta \mathrm{Pg}_{\mathrm{ij}}\right|(j=3)$ \\
\hline G/D12-2a-S22 & 0.10 & 28.59 & 0.94 & - & 4.48 & 0.04 & 2.60 \\
\hline G/D12-2a-R35 & 0.07 & 6.050 & 0.08 & - & 2.27 & 0.10 & 1.31 \\
\hline G/D12-2a-W1 & 0.07 & 3.94 & 0.57 & - & 7.47 & 0.44 & 2.92 \\
\hline G/D12-2a-DAR12 & 0.02 & 36.49 & 1.58 & - & 0.50 & 0.45 & 0.28 \\
\hline G/D12-2a-L59 & 0.17 & 0.00 & 1.87 & - & 8.71 & 2.77 & 2.43 \\
\hline G/D12-1-Y62 & 0.17 & 0.00 & 2.24 & - & 1.31 & 0.51 & 0.69 \\
\hline G/D12-1-Y66 & 5.72 & 153.82 & 1.63 & - & 0.49 & 1.46 & 0.20 \\
\hline G/D12-1-Y67 & 0.23 & 7.78 & 1.22 & - & 0.15 & 0.55 & 0.65 \\
\hline G/D12-1-S22 & 0.08 & 0.00 & 0.12 & - & 2.73 & 2.95 & 0.65 \\
\hline G/D12-1-R35 & 0.25 & 92.59 & 1.14 & - & 4.94 & 2.81 & 0.64 \\
\hline G/D12-1-W1 & 0.11 & 17.35 & 1.63 & - & 0.26 & 2.47 & 0.97 \\
\hline G/D12-1-DAR12 & 0.16 & 219.22 & 2.64 & - & 7.71 & 3.36 & 2.23 \\
\hline G/D12-1-L59 & 0.01 & 66.90 & 0.81 & - & 1.50 & 0.14 & 0.48 \\
\hline Y62-Y66 & 5.89 & 216.32 & 0.61 & 0.0476 & 0.82 & 0.95 & 0.89 \\
\hline Y62-Y67 & 0.06 & 0.00 & 1.02 & 0.0484 & 1.46 & 0.04 & 0.04 \\
\hline Y62-S22 & 0.09 & 0.00 & 2.12 & 0.0484 & 1.42 & 2.44 & 1.34 \\
\hline Y62-R35 & 0.08 & 141.58 & 1.10 & 0.0476 & 3.63 & 2.30 & 0.05 \\
\hline Y62-W1 & 0.06 & 41.94 & 0.61 & 0.1290 & 1.57 & 1.96 & 1.66 \\
\hline Y62-DAR12 & 0.01 & 299.56 & 0.40 & 0.0806 & 6.40 & 2.85 & 1.54 \\
\hline Y62-L59 & 0.16 & 112.72 & 3.05 & 0.0645 & 2.81 & 0.37 & 1.17 \\
\hline Y66-Y67 & 5.95 & 250.73 & 0.41 & 0.0641 & 0.64 & 0.91 & 0.85 \\
\hline Y66-S22 & 5.80 & 130.19 & 1.51 & 0.0645 & 2.24 & 1.49 & 0.45 \\
\hline Y66-R35 & 5.97 & 6.62 & 0.49 & 0.0323 & 4.45 & 1.35 & 0.84 \\
\hline Y66-W1 & 5.83 & 76.84 & 0.00 & 0.1452 & 0.75 & 1.01 & 0.77 \\
\hline Y66-DAR12 & 5.88 & 27.68 & 1.01 & 0.0968 & 7.22 & 1.90 & 2.43 \\
\hline Y66-L59 & 5.73 & 32.73 & 2.44 & 0.0806 & 1.99 & 1.32 & 0.28 \\
\hline Y67-S22 & 0.15 & 33.11 & 1.10 & 0.0968 & 2.88 & 2.40 & 1.30 \\
\hline Y67-R35 & 0.02 & 173.48 & 0.08 & 0.0645 & 5.09 & 2.26 & 0.01 \\
\hline Y67-W1 & 0.12 & 61.54 & 0.41 & 0.1475 & 0.11 & 1.92 & 1.62 \\
\hline Y67-DAR12 & 0.07 & 332.64 & 1.42 & 0.0983 & 7.86 & 2.81 & 1.58 \\
\hline Y67-L59 & 0.22 & 140.38 & 2.03 & 0.0500 & 1.35 & 0.41 & 1.13 \\
\hline S22-R35 & 0.17 & 81.26 & 1.02 & 0.0952 & 2.21 & 0.14 & 1.29 \\
\hline S22-W1 & 0.03 & 17.35 & 1.51 & 0.0847 & 2.99 & 0.48 & 0.32 \\
\hline S22-DAR12 & 0.08 & 159.78 & 2.52 & 0.0984 & 4.98 & 0.41 & 2.88 \\
\hline S22-L59 & 0.07 & 56.29 & 0.93 & 0.1129 & 4.23 & 2.81 & 0.17 \\
\hline R35-W1 & 0.14 & 35.29 & 0.49 & 0.1746 & 5.20 & 0.34 & 1.61 \\
\hline R35-DAR12 & 0.09 & 57.99 & 1.50 & 0.1270 & 2.77 & 0.55 & 1.59 \\
\hline R35-L59 & 0.24 & 0.00 & 1.95 & 0.0806 & 6.44 & 2.67 & 1.12 \\
\hline W1-DAR12 & 0.05 & 146.84 & 1.01 & 0.1803 & 7.97 & 0.89 & 3.20 \\
\hline W1-L59 & 0.10 & 14.03 & 2.44 & 0.1639 & 1.24 & 2.33 & 0.49 \\
\hline DAR12-L59 & 0.15 & 76.51 & 3.45 & 0.1148 & 9.21 & 3.22 & 2.71 \\
\hline
\end{tabular}


$\left|\Delta \mathrm{Fl}_{\mathrm{i}}\right|-\left|\Delta \mathrm{Ph}_{\mathrm{i}}\right|$ or $\left|\Delta \mathrm{Gs}_{\mathrm{i}}\right|-\left|\Delta \mathrm{Ph}_{\mathrm{i}}\right|,\left|\Delta \mathrm{Gs}_{\mathrm{i}}\right|$ versus $\left|\Delta \mathrm{Pg}_{\mathrm{ij}}\right|$, $\left|\Delta \mathrm{Gs}_{\mathrm{i}}\right|$ versus $\left|\Delta \mathrm{Fl}_{\mathrm{i}}\right|,\left|\Delta \mathrm{Ph}_{\mathrm{i}}\right|$ versus $\left|\Delta \mathrm{Pg}_{\mathrm{ij}}\right|,\left|\Delta \mathrm{Fl}_{\mathrm{i}}\right|$ versus $\left|\Delta \mathrm{Pg}_{\mathrm{ij}}\right|,\left|\Delta \mathrm{Fl}_{\mathrm{i}}\right|$ versus $\left|\Delta \mathrm{Ph}_{\mathrm{i}}\right|$.

The pairwise differences by value $i$ where $i$ is genotype number (Table 1 ) are $\left|\Delta \mathrm{Gs}_{\mathrm{i}}\right|$ for genome size, $\left|\Delta \mathrm{Glg}_{\mathrm{i}}\right|$ for genetic distances, $\left|\Delta \mathrm{Fl}_{\mathrm{i}}\right|$ for flavonoids content.

The pairwise linear regression technique was used to carry out procedure of extreme grouping. We think that this technique probably was the only possible way to interpret results. Factor analysis cannot be applied due to the latency of specific environmental factors during the seeds' formation from which genotypes plants have been germinated and which determine a certain adaptation index value. The closest Mantel test only finds cases when the analyzed pa-

Table 5. The example of extreme grouping for pairs of indices $\Delta \mathrm{Ph}_{\mathrm{i}}$ versus $\left|\Delta \mathrm{Gs}_{\mathrm{i}}\right|,\left|\Delta \mathrm{Fl}_{\mathrm{i}}\right|$ versus $\left|\Delta \mathrm{Gs}_{\mathrm{i}}\right|$, $\left|\Delta \mathrm{Fl}_{\mathrm{i}}\right|$ versus $\Delta \mathrm{Ph}_{\mathrm{i}}$ where $\left|\Delta \mathrm{Gs}_{\mathrm{i}}\right|$ are genome size differences, $\Delta \mathrm{Ph}_{\mathrm{i}}$ are leaf length differences, and $\left|\Delta \mathrm{Fl}_{\mathrm{i}}\right|$ are flavonoid content values in leaves differences

\begin{tabular}{|c|c|c|c|c|c|c|}
\hline \multirow{2}{*}{$\Delta \mathrm{i}$} & \multicolumn{2}{|c|}{$\underset{\text { versus }\left|\Delta \mathrm{Gs}_{\mathrm{i}}\right|}{\Delta \mathrm{Ph}_{\mathrm{i}}}$} & \multicolumn{2}{|c|}{$\begin{array}{c}\left|\Delta \mathrm{Fl}_{\mathrm{i}}\right| \\
\text { versus }\left|\Delta \mathrm{Gs}_{\mathrm{i}}\right|\end{array}$} & \multicolumn{2}{|c|}{$\begin{array}{c}\left|\Delta \mathrm{Fl}_{\mathrm{i}}\right| \\
\text { versus } \Delta \mathrm{Ph}_{\mathrm{i}}\end{array}$} \\
\hline & $\mathrm{X}_{\mathrm{i}}^{+}$ & $\mathrm{X}_{\mathrm{i}}^{-}$ & $\mathrm{X}_{\mathrm{i}}^{+}$ & $\mathrm{X}_{\mathrm{i}}^{-}$ & $\mathrm{X}_{\mathrm{i}}^{+}$ & $\mathrm{X}_{\mathrm{i}}^{-}$ \\
\hline G/D4-1-G/D12-2a & 1 & 0 & 1 & 0 & 1 & 0 \\
\hline G/D4-1-G/D12-1 & 1 & 0 & 1 & 0 & 1 & 0 \\
\hline G/D4-1-Y62 & 1 & 0 & 1 & 0 & 1 & 0 \\
\hline G/D4-1-Y66 & 1 & 0 & 0 & 1 & 0 & 1 \\
\hline G/D4-1-Y67 & 1 & 0 & 1 & 0 & 1 & 0 \\
\hline G/D4-1-S22 & 1 & 0 & 1 & 0 & 1 & 0 \\
\hline G/D4-1-R35 & 1 & 0 & 1 & 0 & 0 & 1 \\
\hline G/D4-1-W1 & 1 & 0 & 1 & 0 & 0 & 1 \\
\hline G/D4-1-DAR12 & 0 & 1 & 0 & 1 & 1 & 0 \\
\hline G/D4-1-L59 & 1 & 0 & 0 & 1 & 0 & 1 \\
\hline G/D12-2a-G/D12-1 & 1 & 0 & 1 & 0 & 1 & 0 \\
\hline G/D12-2a-Y62 & 1 & 0 & 1 & 0 & 1 & 0 \\
\hline G/D12-2a-Y66 & 0 & 1 & 0 & 1 & 1 & 0 \\
\hline G/D12-2a-Y67 & 1 & 0 & 1 & 0 & 1 & 0 \\
\hline G/D12-2a-S22 & 1 & 0 & 1 & 0 & 1 & 0 \\
\hline G/D12-2a-R35 & 1 & 0 & 1 & 0 & 1 & 0 \\
\hline G/D12-2a-W1 & 1 & 0 & 1 & 0 & 1 & 0 \\
\hline G/D12-2a-DAR12 & 1 & 0 & 0 & 1 & 0 & 1 \\
\hline G/D12-2a-L59 & 1 & 0 & 0 & 1 & 0 & 1 \\
\hline
\end{tabular}

End of Table 5

\begin{tabular}{|c|c|c|c|c|c|c|}
\hline \multirow{2}{*}{$\Delta \mathrm{i}$} & \multicolumn{2}{|c|}{$\underset{\text { versus }\left|\Delta \mathrm{Gs}_{\mathrm{i}}\right|}{\Delta \mathrm{P}_{\mathrm{i}}}$} & \multicolumn{2}{|c|}{$\begin{array}{c}\left|\Delta \mathrm{F} l_{\mathrm{i}}\right| \\
\text { versus }\left|\Delta \mathrm{Gs}_{\mathrm{i}}\right|\end{array}$} & \multicolumn{2}{|c|}{$\begin{array}{c}\left|\Delta \mathrm{Fl}_{\mathrm{i}}\right| \\
\text { versus } \Delta \mathrm{Ph}_{\mathrm{i}}\end{array}$} \\
\hline & $\mathrm{X}_{\mathrm{i}}^{+}$ & $\mathrm{X}_{\mathrm{i}}^{-}$ & $\mathrm{X}_{\mathrm{i}}^{+}$ & $\mathrm{X}_{\mathrm{i}}^{-}$ & $\mathrm{X}_{\mathrm{i}}^{+}$ & $\mathrm{X}_{\mathrm{i}}^{-}$ \\
\hline G/D12-1-Y62 & 1 & 0 & 0 & 1 & 0 & 1 \\
\hline G/D12-1-Y66 & 1 & 0 & 1 & 0 & 1 & 0 \\
\hline G/D12-1-Y67 & 1 & 0 & 1 & 0 & 1 & 0 \\
\hline G/D12-1-S22 & 1 & 0 & 1 & 0 & 1 & 0 \\
\hline G/D12-1-R35 & 1 & 0 & 1 & 0 & 1 & 0 \\
\hline G/D12-1-W1 & 1 & 0 & 0 & 1 & 0 & 1 \\
\hline G/D12-1-DAR12 & 0 & 1 & 0 & 1 & 1 & 0 \\
\hline G/D12-1-L59 & 1 & 0 & 1 & 0 & 1 & 0 \\
\hline Y62-Y66 & 1 & 0 & 0 & 1 & 0 & 1 \\
\hline Y62-Y67 & 1 & 0 & 1 & 0 & 1 & 0 \\
\hline Y62-S22 & 1 & 0 & 0 & 1 & 0 & 1 \\
\hline Y62-R35 & 1 & 0 & 1 & 0 & 0 & 1 \\
\hline Y62-W1 & 1 & 0 & 1 & 0 & 1 & 0 \\
\hline Y62-DAR12 & 0 & 1 & 1 & 0 & 0 & 1 \\
\hline Y62-L59 & 1 & 0 & 0 & 1 & 1 & 0 \\
\hline Y66-Y67 & 1 & 0 & 0 & 11 & 0 & 1 \\
\hline Y66-S22 & 0 & 1 & 1 & 0 & 1 & 0 \\
\hline Y66-R35 & 0 & 1 & 0 & 1 & 1 & 0 \\
\hline Y66-W1 & 0 & 1 & 0 & 1 & 0 & 1 \\
\hline Y66-DAR12 & 0 & 1 & 0 & 1 & 1 & 0 \\
\hline Y66-L59 & 0 & 1 & 1 & 0 & 0 & 1 \\
\hline Y67-S22 & 1 & 0 & 1 & 0 & 1 & 0 \\
\hline Y67-R35 & 0 & 1 & 1 & 0 & 0 & 1 \\
\hline Y67-W1 & 1 & 0 & 1 & 0 & 0 & 1 \\
\hline Y67-DAR12 & 0 & 1 & 1 & 0 & 0 & 1 \\
\hline Y67-L59 & 1 & 0 & 0 & 1 & 1 & 0 \\
\hline S22-R35 & 1 & 0 & 1 & 0 & 1 & 0 \\
\hline $\mathrm{S} 22-\mathrm{W} 1$ & 1 & 0 & 0 & 1 & 0 & 1 \\
\hline S22-DAR12 & 0 & 1 & 0 & 1 & 1 & 0 \\
\hline S22-L59 & 1 & 0 & 1 & 0 & 1 & 0 \\
\hline R35-W1 & 1 & 0 & 1 & 0 & 1 & 0 \\
\hline R35-DAR12 & 1 & 0 & 0 & 1 & 0 & 1 \\
\hline R35-L59 & 1 & 0 & 0 & 1 & 0 & 1 \\
\hline W1-DAR12 & 1 & 0 & 1 & 0 & 0 & 1 \\
\hline W1-L59 & 1 & 0 & 0 & 1 & 1 & 0 \\
\hline DAR12-L59 & 1 & 0 & 0 & 1 & 0 & 1 \\
\hline
\end{tabular}


rameters are in synchrony. In this context, we compared the differences between genotypes in the measured indices sample series by quantitative differences phase or antiphase (that correspond to synchrony or asynchrony of following adaptation mechanisms in pairwise comparable indices set. Example of the extremal grouping of pairwise spatial differences for indices pairs $\Delta \mathrm{Ph}_{\mathrm{i}}$ versus $\left|\Delta \mathrm{Gs}_{\mathrm{i}}\right|,\left|\Delta \mathrm{Fl}_{\mathrm{i}}\right|$ versus $\left|\Delta \mathrm{Gs}_{\mathrm{i}}\right|,\left|\Delta \mathrm{Fl} \mathrm{l}_{\mathrm{i}}\right|$ versus $\Delta \mathrm{Ph}_{\mathrm{i}}$ and $\left|\Delta \mathrm{Pr}_{\mathrm{i} 1}\right|$ versus $\Delta \mathrm{Ph}_{\mathrm{i}},\left|\Delta \mathrm{Pr}_{\mathrm{i} 1}\right|$ versus $\left|\Delta \mathrm{Fl} \mathrm{i}_{\mathrm{i}}\right|$, $\left|\Delta \operatorname{Pr}_{i 1}\right|$ versus $\left|\Delta \mathrm{Gs}_{\mathrm{i}}\right|$ are shown in Figures 5, 6 .

The pairwise differences by value $i$ where $i$ is genotype number (Table 1 ) are $\left|\Delta \mathrm{Gs}_{\mathrm{i}}\right|$ for genome size, $\left|\Delta \mathrm{Fl}_{\mathrm{i}}\right|$ for flavonoids content, $\Delta \mathrm{Ph}_{\mathrm{i}}$ for leaf length.

The pairwise differences by value $i$ where $i$ is genotype number (Table 1) are $\left|\Delta \mathrm{Gs}_{\mathrm{i}}\right|$ for genome size, $\left|\Delta \mathrm{Fl} \mathrm{i}_{\mathrm{i}}\right|$ for flavonoids content, $\Delta \mathrm{Ph}_{\mathrm{i}}$ for leaf length, $\left|\Delta \mathrm{Pr}_{\mathrm{ik}}\right|$ for pairwise differences of relative content of protective and main proteins in leaves.

Extreme grouping refers to heuristic methods of applied statistical analysis, which need to solve special problems with human participation and those cannot be performed according to a given machine algorithm. Extreme grouping is as follows: on the plane, spatial differences for one parameter and another one for all pairs of experimental genotypes to plot on different axes. The regression line and the coefficient $\mathrm{R}^{2}$ indicate that there is no correlation. Next, the researcher, based on their location, determines the possible configuration of the regression lines passage, which are likely to have positive and negative correlations. Separate point manipulation allows us to divide the points of the spatial difference values into two groups for optimal values of the pairwise linear regression. It should be noted that after the matching into groups with significant correlations, manipulations with each individual doubtfully oriented difference only slightly change the overall picture of grouping.

4. Determination of the matching probability into the positive or negative group for each genotype by number $i$.

The lower index $l$ in the algorithm shown in Figure 4, indicated number of indices pairs, where the indices pairs $\Delta \mathrm{Ph}_{\mathrm{i}}$ versus $\left|\Delta \mathrm{S}_{\mathrm{i}}\right|,\left|\Delta \mathrm{Fl}_{\mathrm{i}}\right|$ versus $\left|\Delta \mathrm{Gs}_{\mathrm{i}}\right|,\left|\Delta \mathrm{Fl}_{\mathrm{i}}\right|$ versus $\Delta \mathrm{Ph}_{\mathrm{i}}$ of $D$. antarctica plant genotypes under in vitro cultivation were assigned value $l=1,2,3$, respectively. Table 5 contains indices pairs presented in the binary approximation form which used to determine the probability matching each genotype (by number $i$ ) into the positive or negative group.

Table 6. The United Latent Index of Adaptability $\left(I_{i}^{q}\left(l_{1}=4\right)\right)$ calculation for the $i$-th genotype Deschampsia antarctica by indices pairs $\Delta \mathrm{Ph}_{\mathrm{i}}$ versus $\left|\Delta \mathrm{Gs}_{\mathrm{i}}\right|\left(I_{i 1}\right),\left|\Delta \mathrm{Fl} \mathrm{l}_{\mathrm{i}}\right|$ versus $\left|\Delta \mathrm{Gs}_{\mathrm{i}}\right|\left(I_{i 2}\right),\left|\Delta \mathrm{Fl} \mathrm{i}_{\mathrm{i}}\right|$ versus $\Delta \mathrm{Ph}_{\mathrm{i}}\left(I_{i 3}\right)$, $\left|\Delta \mathrm{Pr}_{\mathrm{ik}}\right|$ versus $\Delta \mathrm{Ph}_{\mathrm{i}}\left(I_{i 4}\right),\left|\Delta \mathrm{Pr}_{\mathrm{ik}}\right|$ versus $\left|\Delta \mathrm{Fl}_{\mathrm{i}}\right|\left(I_{i 5}\right),\left|\Delta \mathrm{Pr}_{\mathrm{ik}}\right|$ versus $\left|\Delta \mathrm{Gs}_{\mathrm{i}}\right|\left(I_{i 6}\right)$ where $\left|\Delta \mathrm{Gs} \mathrm{s}_{\mathrm{i}}\right|$ are genome size differences, $\Delta \mathrm{Ph}_{\mathrm{i}}$ are leaf length differences, $\left|\Delta \mathrm{F} l_{\mathrm{i}}\right|$ are flavonoid content in leaves differences, $\left|\Delta \mathrm{Pr}_{\mathrm{ik}}\right| \operatorname{are}$ relative content of protective and main proteins in leaves differences

\begin{tabular}{|c|l|c|c|c|c|c|c|c|c|}
\hline $\mathrm{i}$ & $\begin{array}{c}\text { Genotype } \\
\text { name }\end{array}$ & $I_{i 1}$ & $I_{i 2}$ & $I_{i 3}$ & $I_{i}^{q}\left(l_{l}=3\right)$ & $I_{i 4}$ & $I_{i 5}$ & $I_{i 6}$ & $I_{i}^{q}\left(l_{l}=4\right)$ \\
\hline 1 & G/D4-1 & 0.8 & 0.4 & 0.2 & 0.466 & 0.00 & 0.000 & 0.300 & 0.283 \\
2 & G/D12-2a & 0.8 & 0.4 & 0.6 & 0.599 & 0.033 & -0.200 & 0.200 & 0.306 \\
3 & G/D12-1 & 0.8 & 0.4 & 0.6 & 0.599 & 0.033 & 0.167 & 0.233 & 0.372 \\
4 & Y62 & 0.4 & 0.2 & 0.0 & 0.200 & 0.067 & -0.067 & 0.167 & 0.128 \\
5 & Y66 & -0.2 & -0.4 & 0.0 & -0.200 & 0.133 & 0.033 & -0.067 & -0.084 \\
6 & Y67 & 0.6 & 0.6 & 0.2 & 0.466 & 0.000 & 0.033 & 0.167 & 0.267 \\
7 & S22 & 0.6 & 0.4 & 0.6 & 0.533 & 0.067 & -0.067 & 0.267 & 0.311 \\
8 & R35 & 0.6 & 0.4 & 0.0 & 0.333 & 0.067 & 0.233 & 0.333 & 0.272 \\
9 & W1 & 0.6 & 0.2 & -0.4 & 0.133 & 0.033 & -0.100 & 0.167 & 0.083 \\
10 & DAR12 & -0.2 & -0.4 & 0.0 & -0.200 & 0.100 & -0.433 & 0.600 & -0.056 \\
11 & L59 & 0.6 & -0.4 & 0.0 & 0.067 & 0.167 & -0.400 & 0.200 & 0.028 \\
\hline
\end{tabular}




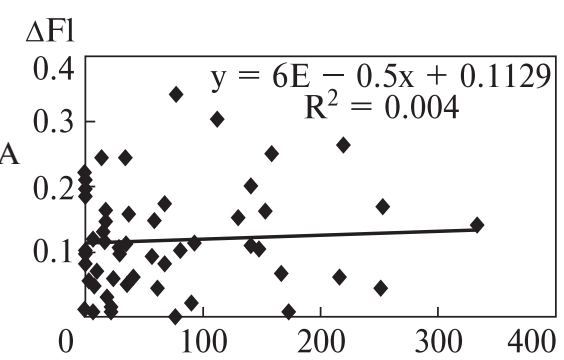

(a)

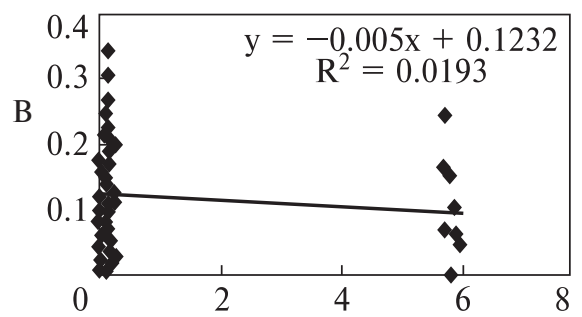

(d)

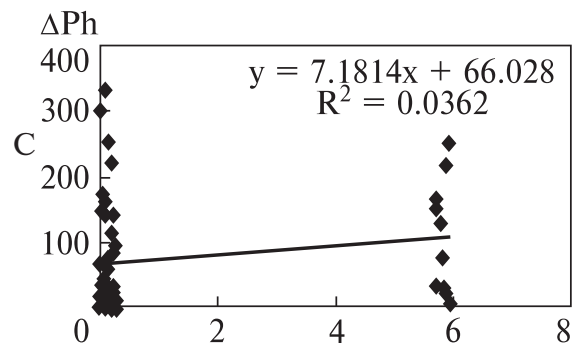

(g)
$\Delta \mathrm{Fl}^{+}$

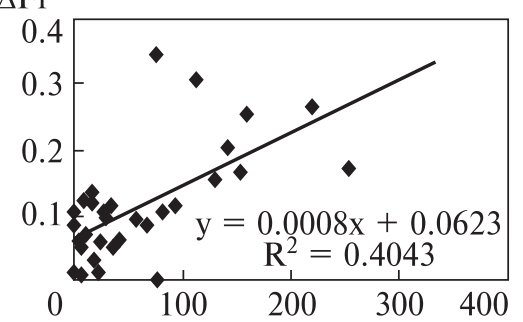

(b)

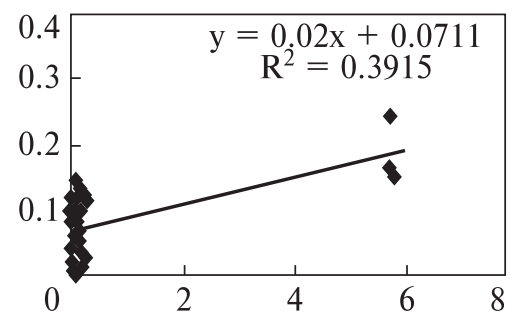

(e)

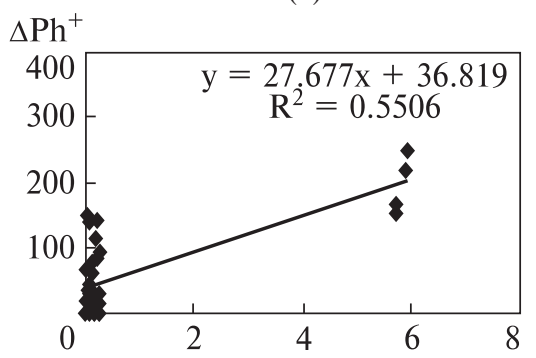

(h)
$\Delta \mathrm{Fl}^{-}$

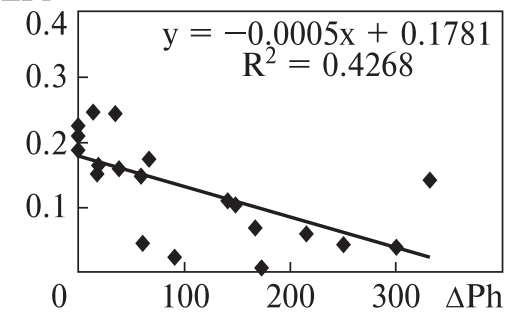

(c)

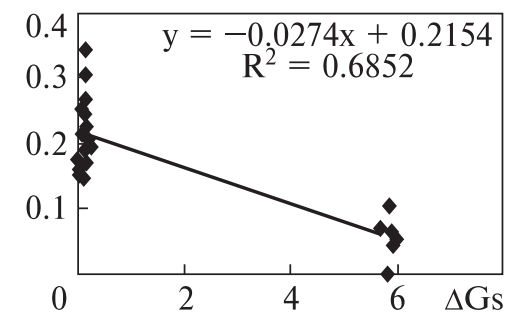

(f)

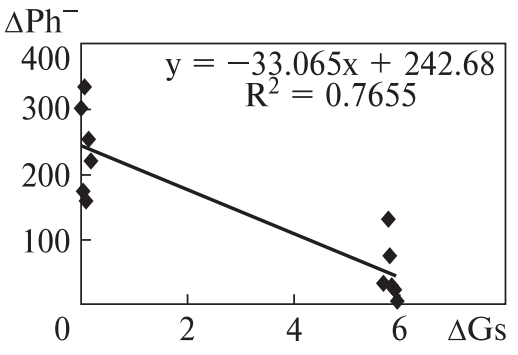

(i)

Figure 5. An example of the extreme grouping method application to $\mathrm{A}$ - a pair of characteristics $\Delta \mathrm{Ph}_{\mathrm{i}}$ versus $\left|\Delta \mathrm{Gs}_{\mathrm{i}}\right|, \mathrm{B}-\left|\Delta \mathrm{Fl}_{\mathrm{i}}\right|$ versus $\left|\Delta \mathrm{Gs}_{\mathrm{i}}\right|, \mathrm{C}-\left|\Delta \mathrm{Fl}_{\mathrm{i}}\right|$ versus $\Delta \mathrm{Ph}_{\mathrm{i}}$ of Deschampsia antarctica plants under cultivation in vitro. Comparison of sets A, B, C: (a), (d), (g) - for all studied variables among all genotypes; (b), (e), (h) - for differences that have a positive correlation between them, obtained by the method of least squares; (c), (f), (i) - for differences that have a negative correlation between these characteristics. The regression equations on the charts by the method of least squares and squares of the corresponding correlation coefficients between values of $\mathrm{A}-$ $\Delta \mathrm{Ph}_{\mathrm{i}}$ versus $\left|\Delta \mathrm{Gs}_{\mathrm{i}}\right|, \mathrm{B}-\left|\Delta \mathrm{Fl}_{\mathrm{i}}\right|$ versus $\left|\Delta \mathrm{Gs}_{\mathrm{i}}\right|, \mathrm{C}-\left|\Delta \mathrm{Fl}_{\mathrm{i}}\right|$ versus $\Delta \mathrm{Ph}_{\mathrm{i}}$. The test value of $\mathrm{R}^{2}$ shown on the charts: (a), (d), (g) $-\mathrm{F}_{1.53}=0.212$, $\mathrm{F}_{1.53}=1.007, \mathrm{~F}_{1.53}=2.120$ respectively (do not exceed the value of the upper $5 \%$ of the F-distribution limit for $\mathrm{N}=55\left(\mathrm{~F}_{1.53}=4.08\right)$ ), (b), (e), (h) $-\mathrm{F}_{1.30}=24.000, \mathrm{~F}_{1.30}=19.290, \mathrm{~F}_{1.41}=50.225$ respectively, and (c), (f), (i) $-\mathrm{F}_{1.21}=11.382, \mathrm{~F}_{1.21}=45.717, \mathrm{~F}_{1.10}=32.640$ respectively (exceeds the upper $5 \%$ of the F-distribution limit for $\mathrm{N}=32\left(\mathrm{~F}_{1,30}=4.17\right), \mathrm{N}=32\left(\mathrm{~F}_{1,30}=4.17\right), \mathrm{N}=43\left(\mathrm{~F}_{1,41}=4.08\right)$ and $\mathrm{N}=23\left(\mathrm{~F}_{1,21}=4.32\right), \mathrm{N}=23\left(\mathrm{~F}_{1,21}=4.32\right), \mathrm{N}=12\left(\mathrm{~F}_{1,10}=4.96\right)$ respectively $)$. This means there is no linear dependence in cases (a), (d), (g) and the presence of linear dependence in cases (b), (e), (h) and (c), (f), (i)

The parts of matches to the positive and negative groups $\mathrm{X}_{\mathrm{i}}^{+}$and $\mathrm{X}_{\mathrm{i}}^{-}$have been determined for each value $i$. The example is presented in Table 5. The number genotypes was $i_{\max }=\mathrm{n}$ (in the considered case $\mathrm{n}=11$ ). The maximum number of points was $n-1$ on the plot. To determine the total matching probability to $\mathrm{X}^{+}{ }_{\mathrm{i}}$ or $\mathrm{X}_{\mathrm{i}}^{-}$for indices pairs $\Delta \mathrm{Ph}_{\mathrm{i}}$ versus $\left|\Delta \mathrm{Gs}_{\mathrm{i}}\right|,\left|\Delta \mathrm{Fl}_{\mathrm{i}}\right|$ versus $\left|\Delta \mathrm{Gs}_{\mathrm{i}}\right|,\left|\Delta \mathrm{Fl}_{\mathrm{i}}\right|$ versus $\Delta \mathrm{Ph}_{\mathrm{i}}$ formula (1) was applied for each value $l=1,2,3$ :
$\Delta \mathrm{Ph}_{\mathrm{i}}$ versus $\left|\Delta \mathrm{Gs}_{\mathrm{i}}\right|,\left|\Delta \mathrm{Pr}_{\mathrm{ik}}\right|$ versus $\left|\Delta \mathrm{Gs}_{\mathrm{i}}\right|,\left|\Delta \mathrm{Pr}_{\mathrm{ik}}\right|$ versus $\Delta \mathrm{Ph}_{\mathrm{i}}$

$$
X_{i l}=\frac{1}{n-1}\left(X_{i l}^{+}-X_{i l}^{-}\right) \text {for } l=1,2,3 .
$$

When we add additional datasets such as $\left|\Delta \operatorname{Pr}_{\mathrm{ik}}\right|$ or $\left|\Delta \mathrm{Glg}_{\mathrm{i}}\right|$ or $\left|\Delta \mathrm{Pg}_{\mathrm{ij}}\right|(l=4)$, the formula (2) is used.

$$
X_{i l}=\frac{1}{n-1} \sum_{p}\left(X_{i p}^{+}-X_{i p}^{-}\right) \text {for } l=4, p=\mathrm{k} \text { or } p=\mathrm{j} .
$$




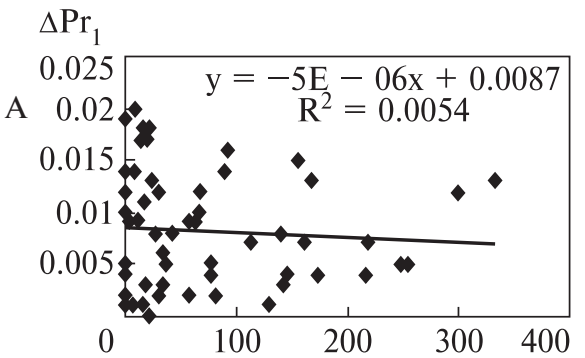

(a)

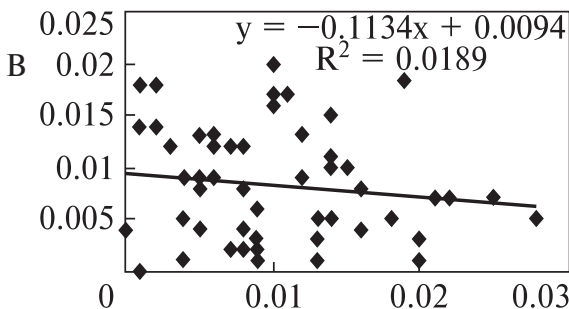

(d)

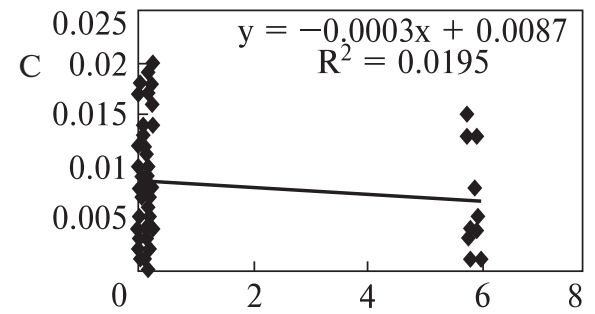

(g)

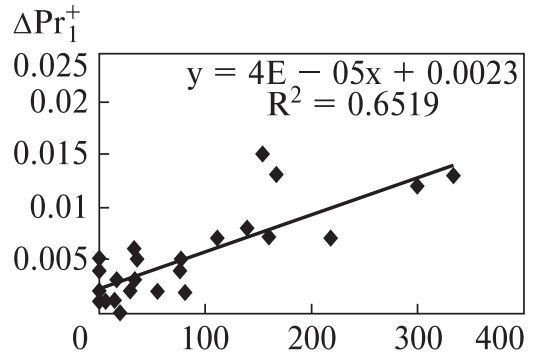

(b)

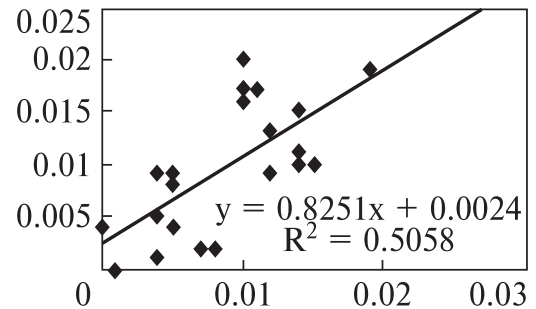

(e)

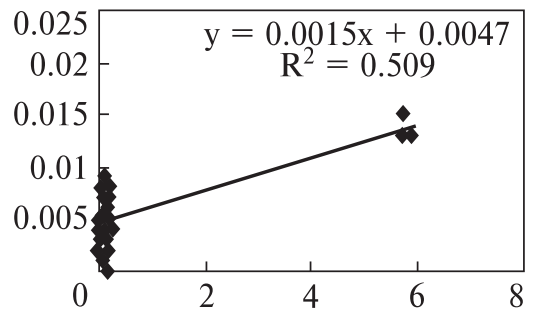

(h)

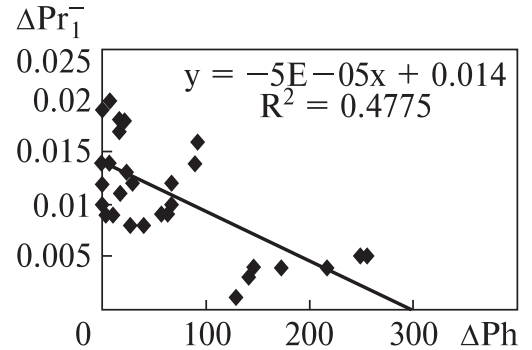

(c)

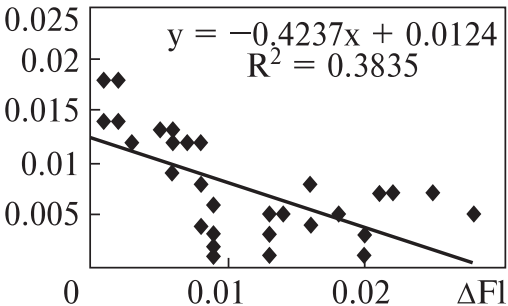

(f)

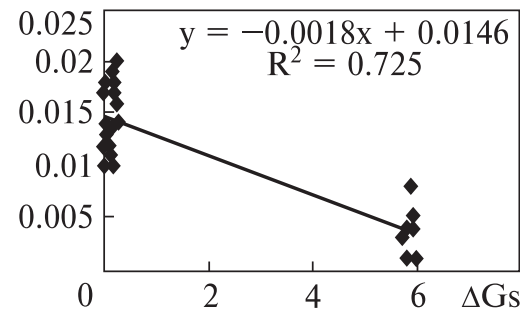

(i)

Figure 6. An example of the extreme grouping method application to $\mathrm{A}$ - a pair of characteristics $\left|\Delta \mathrm{Pr}_{\mathrm{i} 1}\right| \operatorname{versus} \Delta \mathrm{Ph}_{\mathrm{i}}, \mathrm{B}-\left|\Delta \mathrm{Pr}_{\mathrm{i} 1}\right|$ versus $\left|\Delta \mathrm{Fl}_{\mathrm{i}}\right|, \mathrm{C}-\left|\Delta \mathrm{Pr}_{\mathrm{i} 1}\right|$ versus $\left|\Delta \mathrm{Gs}_{\mathrm{i}}\right|$ of Deschampsia antarctica plants under cultivation in vitro. Comparison of sets $\mathrm{A}, \mathrm{B}, \mathrm{C}$ : (a), (d), (g) - for all studied variables among all genotypes; (b), (e), (h) - for differences that have a positive correlation between them, obtained by the method of least squares; (c), (f), (i) - for differences that have a negative correlation between these characteristics. The regression equations on the charts by the method of least squares and squares of the corresponding correlation coefficients between values of $\mathrm{A}-\left|\Delta \mathrm{Pr}_{\mathrm{i} 1}\right|$ versus $\Delta \mathrm{Ph}_{\mathrm{i}}, \mathrm{B}-\left|\Delta \mathrm{Pr}_{\mathrm{i} 1}\right|$ versus $\left|\Delta \mathrm{Fl}_{\mathrm{i}}\right|, \mathrm{C}-\left|\Delta \mathrm{Pr}_{\mathrm{i} 1}\right|$ versus $\left|\Delta \mathrm{Gs}_{\mathrm{i}}\right|$. The test value of $\mathrm{R}^{2}$ shown on the charts: (a), (d), $(\mathrm{g})-\mathrm{F}_{1,53}=0.265, \mathrm{~F}_{1,53}=1.113, \mathrm{~F}_{1,53}=1.060$ respectively (do not exceed the value of the upper $5 \%$ of the F-distribution limit for $\left.\mathrm{N}=55\left(\mathrm{~F}_{1.53}=4.08\right)\right)$, (b), (e), (h) $-\mathrm{F}_{1,23}=43.079, \mathrm{~F}_{1,21}=18.879$, $\mathrm{F}_{1,28}=29.036$ respectively, and (c), (f), (i) $-\mathrm{F}_{1,28}=25.592, \mathrm{~F}_{1,30}=17.280, \mathrm{~F}_{1,23}=60.628$ respectively (exceeds the upper $5 \%$ of the $\mathrm{F}$-distribution limit for $\mathrm{N}=25$ $\left(\mathrm{F}_{1,23}=4.28\right), \mathrm{N}=23\left(\mathrm{~F}_{1,21}=4.32\right), \mathrm{N}=30\left(\mathrm{~F}_{1,28}=4.20\right)$ and $\mathrm{N}=30\left(\mathrm{~F}_{1,28}=4.20\right), \mathrm{N}=32\left(\mathrm{~F}_{1,30}=4.17\right), \mathrm{N}=25\left(\mathrm{~F}_{1,23}=4.28\right)$ respectively). This means there is no linear dependence in cases (a), (d), (g) and the presence of linear dependence in cases (b), (e), (h) and (c), (f), (i)

\section{Determination of the normalization factor for each data set. \\ For the simplest case (Tables 4 and 5, Fig. 5), the normalization factors are equal to one. When we add additional datasets such as $\left|\Delta \operatorname{Pr}_{\mathrm{ik}}\right|\left(\right.$ Fig. 6) or $\left|\Delta \operatorname{Pg}_{\mathrm{ij}}\right|(l=4)$, each indices pair $\left|\Delta \mathrm{Pr}_{\mathrm{ik}}\right|$ versus $\Delta \mathrm{Ph}_{\mathrm{i}},\left|\Delta \mathrm{Pr}_{\mathrm{ik}}\right|$ versus $\left|\Delta \mathrm{Fl}_{\mathrm{i}}\right|$, $\left|\Delta \operatorname{Pr}_{\mathrm{ik}}\right|$ versus $\left|\Delta \mathrm{Gs}_{\mathrm{i}}\right|(k=6)$ or $\left|\Delta \mathrm{Pg}_{\mathrm{ij}}\right|$ versus $\Delta \mathrm{Ph}_{\mathrm{i}},\left|\Delta \mathrm{Pg}_{\mathrm{ij}}\right|$ versus $\left|\Delta \mathrm{Fl}_{\mathrm{i}}\right|,\left|\Delta \mathrm{Pg}_{\mathrm{ij}}\right|$ versus $\left|\Delta \mathrm{Gs}_{\mathrm{i}}\right|(j=3)$ had a different total number of points on the planes compared to the}

source three pairs of indices $\Delta \mathrm{Ph}_{\mathrm{i}}$ versus $\left|\Delta \mathrm{Gs}_{\mathrm{i}}\right|,|\Delta \mathrm{F}|_{\mathrm{i}} \mid$ versus $\left|\Delta \mathrm{Gs}_{\mathrm{i}}\right|,\left|\Delta \mathrm{Fl}_{\mathrm{i}}\right|$ versus $\Delta \mathrm{Ph}_{\mathrm{i}}$, which were subject to extreme grouping, so the determination of normalization factors was performed for them by the following formulas. Normalization factor for the fourth added index $\left|\Delta \operatorname{Pr}_{i \mathrm{i}}\right|($ Figs. 5, 6) had quantity of pair chart $\mathrm{k}=6$ for $\left|\Delta \operatorname{Pr}_{i \mathrm{ik}}\right|$ versus $\Delta \mathrm{Ph}_{\mathrm{i}},\left|\Delta \mathrm{Pr}_{\mathrm{ik}}\right|$ versus $\left|\Delta \mathrm{Fl}_{\mathrm{i}}\right|,\left|\Delta \mathrm{Pr}_{\mathrm{ik}}\right|$ versus $\left|\Delta \mathrm{Gs}_{\mathrm{i}}\right|$ and was determined by the formula:

$$
L_{i k l}=1 / k(l=4), L_{i 64}=0.167 .
$$




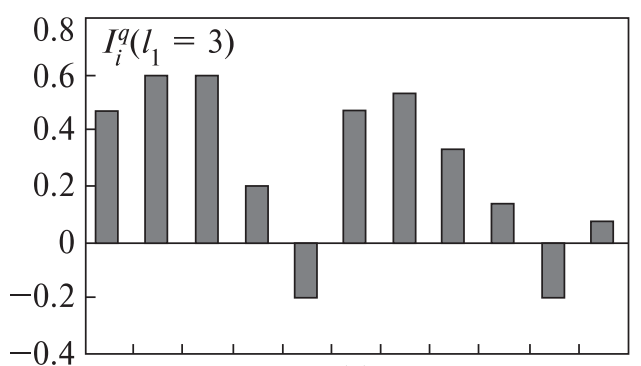

(a)

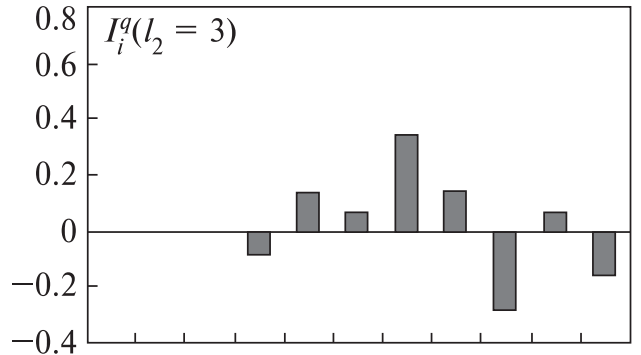

(c)

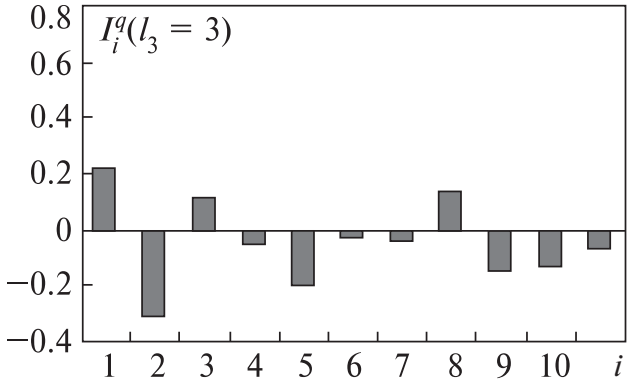

(e)

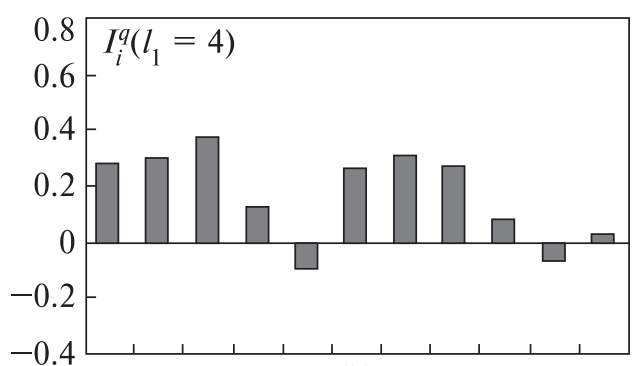

(b)

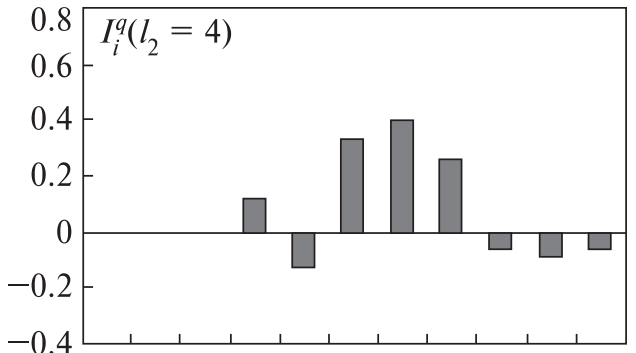

(d)

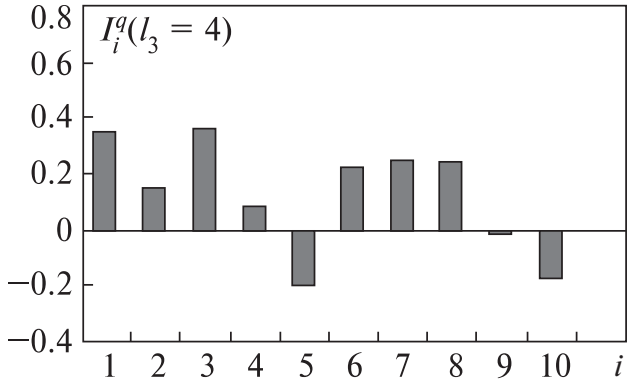

(f)

Figure 7. United Quality Latent Indices of Adaptability for studied Deschampsia antarctica genotypes based on sources pairwise data sets: (a) - three pairwise data sets $\left(l_{1}=3\right) \Delta \mathrm{Ph}_{\mathrm{i}}$ versus $\left|\Delta \mathrm{Gs}_{\mathrm{i}}\right|,\left|\Delta \mathrm{Fl}_{\mathrm{j}}\right|$ versus $\left|\Delta \mathrm{Gs}_{\mathrm{i}}\right|,\left|\Delta \mathrm{Fl}_{\mathrm{i}}\right|$ versus $\Delta \mathrm{Ph}_{\mathrm{i}}$ for eleven genotypes; (b) four pairwise data sets $\left(l_{1}=4\right): \Delta \mathrm{Ph}_{\mathrm{i}}$ versus $\left|\Delta \mathrm{Gs}_{\mathrm{i}}\right|,\left|\Delta \mathrm{Fl}_{\mathrm{i}}\right|$ versus $\left|\Delta \mathrm{Gs}_{\mathrm{i}}\right|,\left|\Delta \mathrm{Fl}_{\mathrm{i}}\right|$ versus $\Delta \mathrm{Ph}_{\mathrm{i}},\left|\Delta \mathrm{Pr}_{\mathrm{ik}}\right|$ versus $\Delta \mathrm{Ph}_{\mathrm{i}},\left|\Delta \mathrm{Pr}_{\mathrm{ik}}\right|$ versus $\left|\Delta \mathrm{Fl}_{\mathrm{i}}\right|$, $\left|\Delta \mathrm{Pr}_{\mathrm{ik}}\right|$ versus $\left|\Delta \mathrm{Gs}_{\mathrm{i}}\right|$ for eleven genotypes; (c) - three pairwise data sets $\left(l_{2}=3\right) \Delta \mathrm{Ph}_{\mathrm{i}}$ versus $\left|\Delta \mathrm{Glg}_{\mathrm{i}}\right|,\left|\Delta \mathrm{Fl}_{\mathrm{i}}\right|$ versus $\left|\Delta \mathrm{Glg}_{\mathrm{i}}\right|,\left|\Delta \mathrm{Fl}_{\mathrm{i}}\right|$ versus $\Delta \mathrm{Ph}_{\mathrm{i}}$ for eigth genotypes; (d) - four pairwise data sets $\left(\mathrm{l}_{2}=4\right) \Delta \mathrm{Ph}_{\mathrm{i}}$ versus $\left|\Delta \mathrm{Gs}_{\mathrm{i}}\right|,\left|\Delta \mathrm{Fl}_{\mathrm{i}}\right|$ versus $\left|\Delta \mathrm{Gs}_{\mathrm{i}}\right|,\left|\Delta \mathrm{Fl}_{\mathrm{i}}\right|$ versus $\Delta \mathrm{Ph}_{\mathrm{i}}, \Delta \mathrm{Ph}_{\mathrm{i}}$ versus $\left|\Delta \mathrm{Glg}_{\mathrm{i}}\right|,\left|\Delta \mathrm{Fl}_{\mathrm{i}}\right|$ versus $\left|\Delta \mathrm{Glg}_{\mathrm{i}},\right| \Delta \mathrm{Fl}_{\mathrm{i}} \mid$ versus $\Delta \mathrm{Ph}_{\mathrm{i}},\left|\Delta \mathrm{Glg}_{\mathrm{i}}\right|$ versus $\left|\Delta \mathrm{Gs}_{\mathrm{i}}\right|$ for eigth genotypes; (e) - three pairwise data sets $\left(l_{3}=3\right) \Delta \mathrm{Ph}_{\mathrm{i}}$ versus $\left|\Delta \mathrm{Pg}_{\mathrm{ij}}\right|,\left|\Delta \mathrm{Fl}_{\mathrm{i}}\right|$ versus $\left|\Delta \mathrm{Pg}_{\mathrm{ij}}\right|,\left|\Delta \mathrm{Fl}_{\mathrm{i}}\right|$ versus $\Delta \mathrm{Ph}_{\mathrm{i}}$ for eleven genotypes; (f) - four pairwise data sets $\left(l_{3}=4\right) \Delta \mathrm{Ph}_{\mathrm{i}} \mathrm{versus}\left|\Delta \mathrm{Gs}_{\mathrm{i}}\right|,\left|\Delta \mathrm{Fl}_{\mathrm{i}}\right|$ versus $\left|\Delta \mathrm{Gs}_{\mathrm{i}}\right|,\left|\Delta \mathrm{Fl}_{\mathrm{i}}\right|$ versus $\Delta \mathrm{Ph}_{\mathrm{i}}, \Delta \mathrm{Ph}_{\mathrm{i}}$ versus $\left|\Delta \mathrm{Pg}_{\mathrm{ij}}\right|,\left|\Delta \mathrm{Fl}_{\mathrm{i}}\right|$ versus $\left|\Delta \mathrm{Pg}_{\mathrm{ij}}\right|,\left|\Delta \mathrm{Fl}_{\mathrm{i}}\right|$ versus $\Delta \mathrm{Ph}_{\mathrm{i}},\left|\Delta \mathrm{Pg}_{\mathrm{ij}}\right|$ versus $\left|\Delta \mathrm{Gs}_{\mathrm{i}}\right|$ for eleven genotypes

Normalization factor for the fourth added index $\left|\Delta \mathrm{Pg}_{\mathrm{ij}}\right|$ had quantity of pair chart $j=3$ for $\left|\Delta \mathrm{Pg}_{\mathrm{ij}}\right|$ versus $\Delta \mathrm{Ph}_{\mathrm{i}},\left|\Delta \mathrm{Pg}_{\mathrm{ij}}\right|$ versus $\left|\Delta \mathrm{Fl}_{\mathrm{i}}\right|,\left|\Delta \mathrm{Pg}_{\mathrm{ij}}\right|$ versus $\left|\Delta \mathrm{Gs}_{\mathrm{i}}\right|$ and was determined by formula:

$$
L_{i j l}=1 / j(l=4), L_{i 34}=0.333 .
$$

6. Determination of United Quality Indices for each pair of differences sets for the $i$-th genotype.

United Quality Indices for each pair of indices was denoted by $I_{i 1}, I_{i 2}, I_{i 3}$ for $\Delta \mathrm{Ph}_{\mathrm{i}}$ versus $\left|\Delta \mathrm{Gs}_{\mathrm{i}}\right|,\left|\Delta \mathrm{Fl}_{\mathrm{i}}\right|$ ver- sus $\left|\Delta \mathrm{Gs}_{\mathrm{i}}\right|,\left|\Delta \mathrm{Fl}_{\mathrm{i}}\right|$ versus $\Delta \mathrm{Ph}_{\mathrm{i}}$, respectively. The formula for United Quality Indices was written based on formula (1):

$$
I_{i l}^{q}=L_{i l} \times X_{i l}=\frac{1}{(n-1)}\left(X_{l i}^{+}-X_{l i}^{-}\right) \text {for } l=1,2,3 .
$$

For the extended variant, we denoted the United Quality Index for each pair of indices $I_{i l}, I_{i 2}, I_{i 3}, I_{i q}, I_{i 5}$, $I_{i 6}$ for $\Delta \mathrm{Ph}_{\mathrm{i}}$ versus $\left|\Delta \mathrm{Gs}_{\mathrm{i}}\right|,\left|\Delta \mathrm{Fl}_{\mathrm{i}}\right|$ versus $\left|\Delta \mathrm{Gs}_{\mathrm{i}}\right|,\left|\Delta \mathrm{Fl}_{\mathrm{i}}\right|$ versus $\Delta \mathrm{Ph}_{\mathrm{i}}$ and $\left|\Delta \mathrm{Pr}_{\mathrm{ik}}\right|$ versus $\Delta \mathrm{Ph}_{\mathrm{i}},\left|\Delta \mathrm{Pr}_{\mathrm{ik}}\right|$ versus $\left|\Delta \mathrm{Fl}_{\mathrm{i}}\right|,\left|\Delta \mathrm{Pr}_{\mathrm{ik}}\right|$ 
versus $\left|\Delta \mathrm{Gs}_{\mathrm{i}}\right|$ or $\left|\Delta \mathrm{Pg}_{\mathrm{ij}}\right|$ versus $\Delta \mathrm{Ph}_{\mathrm{i}},\left|\Delta \mathrm{Pg}_{\mathrm{ij}}\right|$ versus $\left|\Delta \mathrm{Fl}_{\mathrm{i}}\right|$, $\left|\Delta \mathrm{Pg}_{\mathrm{ij}}\right|$ versus $\left|\Delta \mathrm{Gs}_{\mathrm{i}}\right|$ correspondingly. The formula for intermediate United Quality Indices was written based on formula (2):

$$
\begin{gathered}
I_{i l}^{q}=L_{i p l} \times X_{i p l}=\frac{1}{p(n-1)} \sum_{p}\left(X_{i p l}^{+}-X_{i p l}^{-}\right) \\
\text {for } l=4, p=\mathrm{k} \text { or } p=\mathrm{j},
\end{gathered}
$$

where $X^{+}{ }_{i l}, X^{+}{ }_{i p l}$ are the matches to the positive group, $X^{-}{ }_{i l}, X^{-}{ }_{i p l}$ are the matches to the negative group, $X_{i l}$, $X_{i p l}$ are summary $i$-th genotype matches probability for each pair of indices $\Delta \mathrm{Ph}_{\mathrm{i}}$ versus $\left|\Delta \mathrm{Gs}_{\mathrm{i}}\right|,\left|\Delta \mathrm{Fl} \mathrm{l}_{\mathrm{i}}\right|$ versus $\left|\Delta \mathrm{Gs}_{\mathrm{i}}\right|,\left|\Delta \mathrm{Fl}_{\mathrm{i}}\right|$ versus $\Delta \mathrm{Ph}_{\mathrm{i}}$ and $\left|\Delta \mathrm{Pr}_{\mathrm{ik}}\right|$ versus $\Delta \mathrm{Ph}_{\mathrm{i}},\left|\Delta \mathrm{Pr}_{\mathrm{ik}}\right|$ versus $\left|\Delta \mathrm{Fl}_{\mathrm{i}}\right|,\left|\Delta \mathrm{Pr}_{\mathrm{ik}}\right|$ versus $\left|\Delta \mathrm{Gs}_{\mathrm{i}}\right|$ or $\left|\Delta \mathrm{Pg}_{\mathrm{ij}}\right|$ versus $\Delta \mathrm{Ph}_{\mathrm{i}}$, $\left|\Delta \mathrm{Pg}_{\mathrm{ij}}\right|$ versus $\left|\Delta \mathrm{Fl}_{\mathrm{i}}\right|,\left|\Delta \mathrm{Pg}_{\mathrm{ij}}\right|$ versus $\left|\Delta \mathrm{Gs}_{\mathrm{i}}\right|$ accordingly, $\mathrm{L}_{i p l}$ is the normalization factor for each indices pair of indices above.

\section{Determination of the United Quality Latent Index} for $i$-th genotype.

The final formula (5) for determining the United Latent Quality Index of Adaptability for $i$-th genotype look like this:

$$
I_{i}^{q}=\frac{1}{3} \sum_{l=1}^{3} I_{i l}^{q} \text { for } l=3 .
$$

Table 7. United Quality Latent Indices of Adaptability $\left(I_{i}{ }_{i}\left(l_{2}=3\right)\right)$ for eight studied Deschampsia antarctica genotypes based on three sources of data $\left(l_{2}=3\right)$ : $\Delta \mathrm{Ph}_{\mathrm{i}}$ versus $\left|\Delta \mathrm{Glg}_{\mathrm{i}}\right|\left(I_{i 1}\right),\left|\Delta \mathrm{Fl}_{\mathrm{i}}\right|$ versus $\left|\Delta \mathrm{Glg}_{\mathrm{i}}\right|\left(I_{i 2}\right),\left|\Delta \mathrm{Fl}_{\mathrm{i}}\right|$ versus $\Delta \mathrm{Ph}_{\mathrm{i}}\left(I_{i 3}\right)$ where $\Delta \mathrm{Ph}_{\mathrm{i}}$ are leaf length differences, $\left|\Delta \mathrm{Fl}_{\mathrm{i}}\right|$ are flavonoid content in leaves differences and $\left|\Delta \mathrm{Glg}_{\mathrm{i}}\right|$ are genetic distances according to Jacquard

\begin{tabular}{|r|c|c|c|c|c|}
\hline $\mathrm{i}$ & Genotype name & $I_{i 1}$ & $I_{i 2}$ & $I_{i 3}$ & $I_{i}^{q}\left(I_{2}=3\right)$ \\
\hline 1 & G/D4-1 & - & - & - & - \\
2 & G/D12-2a & - & - & - & - \\
3 & G/D12-1 & - & - & - & - \\
4 & Y62 & -0.33 & 0.11 & 0 & -0.073 \\
5 & Y66 & 0.11 & 0.33 & 0 & 0.147 \\
6 & Y67 & -0.33 & 0.33 & 0.2 & 0.067 \\
7 & S22 & 0.33 & 0.11 & 0.6 & 0.346 \\
8 & R35 & 0.11 & 0.33 & 0 & 0.147 \\
9 & W1 & -0.11 & -0.33 & -0.4 & -0.280 \\
10 & DAR12 & 0.11 & 0.11 & 0 & 0.073 \\
11 & L59 & 0.11 & 0.56 & 0 & -0.150 \\
\hline
\end{tabular}

Since it is planned to increase the number of studied indices sets of genotypes in vitro conditions in this research it should be taken into account the number of combinations of $l$ elements taken two at a time by the formula:

$$
C_{l}^{2}=\frac{l !}{2 !(l-2) !}
$$

Then for the most general case, formula (5) will look like this:

$$
I_{i}^{q}=\frac{2 !(l-2) !}{l !} \sum_{l} I_{i l}^{q} .
$$

The results for the above example of eleven $D$. antarctica genotypes is presented in Table 6 and Figure 7.

The pairwise differences by value i where $i$ is genotype number (Table 1) are $\left|\Delta \mathrm{Gs}_{\mathrm{i}}\right|$ for genome size, $\left|\Delta \mathrm{Glg}_{\mathrm{i}}\right|$ for genetic distances, $\left|\Delta F l_{i}\right|$ for flavonoids content, $\Delta \mathrm{Ph}_{\mathrm{i}}$ for leaf length, $\left|\Delta \mathrm{Pr}_{\mathrm{ik}}\right|$ for pairwise differences of relative content of protective and main proteins in leaves, $\left|\Delta \mathrm{Pg}_{\mathrm{ij}}\right|$ for photosynthetic pigments.

An example of UQLI $\left(I^{q}\right)$ for samples of 11 genotypes cultivated in vitro is shown in Figures 7a, 7b. The algorithm described above allows increasing the abstraction level of the genotypes samples description at the integral index level, as well as building probabilistic interaction graphs of individual adaptability indices (correlation models). It is interesting that the comparison of two series of data UQLI $\left(I^{q}{ }_{i}\right) 11$ genotypes for variants with $l_{1}=3$ and $l_{1}=4$ (by addition of the data block $\left.\left|\Delta \operatorname{Pr}_{\mathrm{ik}}\right|\right)$ has shown a correlation with $\mathrm{R}=0.982\left(\mathrm{~F}_{1,9}=\right.$ $=244.52$ compared to the tabular value of $5 \%$ limits for $\mathrm{N}=11 \mathrm{~F}_{1,9}=5.12$ ). This means that the UQLI $\left(I_{i}^{q}\right)$ profile when adding a data block $\left|\Delta \mathrm{Pr}_{\mathrm{ik}}\right|$ was saved very well (correlation is statistical confidently), and no $I^{q}{ }_{i}$ change its sign. In further studies, we added each one data block to the main block with $l_{1}=3$ to see if the addition of this block differences the UQLI $\left(I_{i}^{q}\right)$ profile for the sample of 11 or 8 genotypes.

We reiterate that UQLI $\left(I^{q}\right)$ is not a constant value and as a quality index depends on many internal and external factors. Therefore, the cultivation of plants in vitro allows to some extent to capture external factors and focus on internal ones. Usually, the successive refinement of a formula is carried out by stepwise addition of components and assessment of whether 
G/D4-1

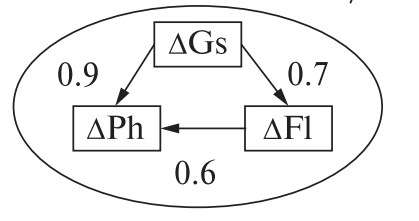

Y62
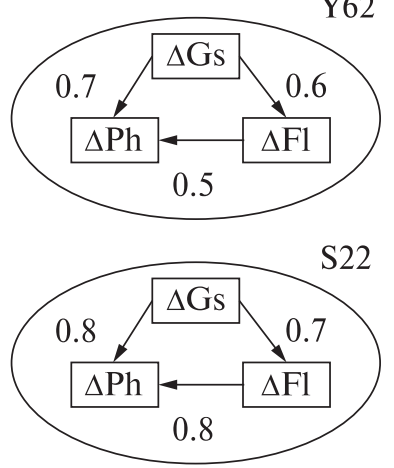

DAR12

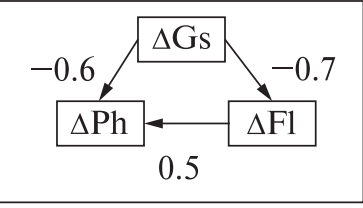

G/D12-2a
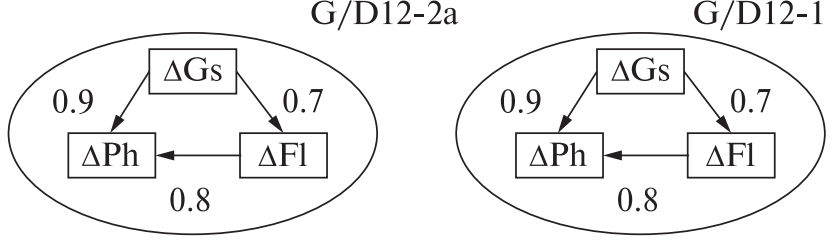

Y66
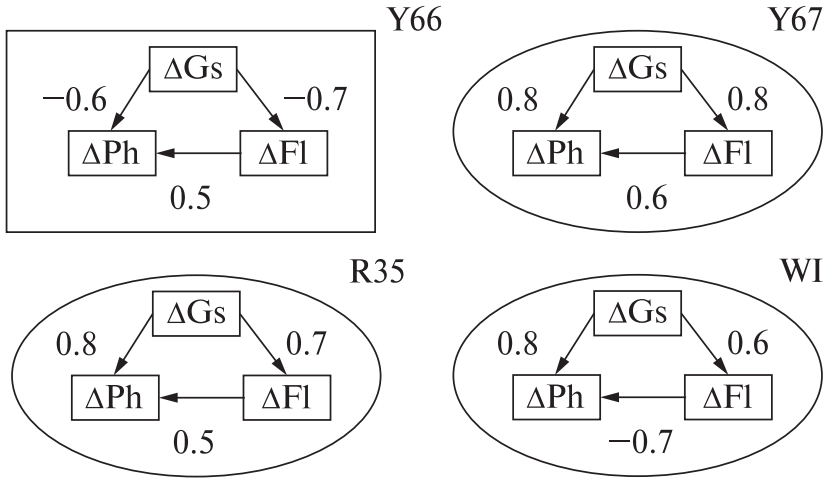

L59

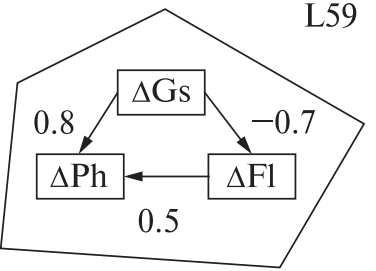

Figure 8. The triangle graphs of probability relations: $\left|\Delta \mathrm{Gs}_{\mathrm{i}}\right| \rightarrow \Delta \mathrm{Ph}_{\mathrm{i}},\left|\Delta \mathrm{Gs}_{\mathrm{i}}\right| \rightarrow\left|\Delta \mathrm{Fl}_{\mathrm{i}}\right|,\left|\Delta \mathrm{Fl}_{\mathrm{i}}\right| \rightarrow \Delta \mathrm{Ph}_{\mathrm{i}}$ for eleven studied genotypes Deschampsia antarctica in vitro based on three data sets $\left(l_{1}=3\right)$ ('genetic size' graphs). Numbers indicate the synchronous $(+)$ or asynchronous (-) processes probability for each studied genotype described by comparable data sets. The genotype graphs (graphs of diploids) with positive probability relations are outlined by ovals, graphs of genotypes with two negative relations $\left(\left|\Delta \mathrm{Gs}_{\mathrm{i}}\right| \rightarrow \Delta \mathrm{Ph}_{\mathrm{i}}\left|\Delta \mathrm{Gs}_{\mathrm{i}}\right| \rightarrow \mid \Delta \mathrm{Fl}_{\mathrm{i}}\right)$ are outlined by rectangles (there are diploid with B-chromosomes DAR12 and hypotriploid genotype Y66), diploid genotype L59, which has only one negative probability relation $\left(\left|\Delta \mathrm{Gs}_{\mathrm{i}}\right| \rightarrow\left|\Delta \mathrm{Fl}_{\mathrm{i}}\right|\right)$ is outlined by pentagon (Parnikoza et al., 2017). The pairwise differences by value $i$ where $i$ is genotype number (Table 1 ) are $\left|\Delta \mathrm{Gs}_{\mathrm{i}}\right|$ for genome size, $\left|\Delta \mathrm{Fl}_{\mathrm{i}}\right|$ for flavonoids content, $\Delta \mathrm{Ph}_{\mathrm{i}}$ for leaf length

they are values of the second order of smallness or not. In our case, such a straightforward approach will not work because each component of the UQLI $\left(I^{q}{ }_{i}\right)$ is important. Therefore, we will assess the significance of each added to the $I_{i}^{q}$ component, which changes the $I_{i}^{q}$ sign for any genotype provided by the addition of this new component. Therefore, we will not yet consider the data set $\left|\Delta \mathrm{Pr}_{\mathrm{ik}}\right|$.

To take into account differences not only at the genome size level but also at the nucleotide sequences one we formed not only a set of $\Delta \mathrm{Ph}_{\mathrm{i}}$ versus $\left|\Delta \mathrm{Gs}_{\mathrm{i}}\right|$, $\left|\Delta \mathrm{Fl}_{\mathrm{i}}\right|$ versus $\left|\Delta \mathrm{Gs}_{\mathrm{i}}\right|,\left|\Delta \mathrm{F} l_{\mathrm{i}}\right|$ versus $\Delta \mathrm{Ph}_{\mathrm{i}}\left(l_{1}=3\right)$ but also the set $\left|\Delta \mathrm{Glg}_{\mathrm{i}}\right|$ versus $\Delta \mathrm{Ph}_{\mathrm{i}},\left|\Delta \mathrm{Glg}_{\mathrm{i}}\right|$ versus $\left|\Delta \mathrm{Fl}_{\mathrm{i}}\right|,\left|\Delta \mathrm{Fl}_{\mathrm{i}}\right|$ versus $\Delta \mathrm{Ph}_{\mathrm{i}}\left(l_{2}=3\right)$ where $\left|\Delta \mathrm{Glg}_{\mathrm{j}}\right|$ is a genetic dis- tances set by ISSR and IRAP primers in eight studied D. antarctica genotypes (Table 7).

UQLI $\left(I^{q}\right)$ values for samples of eight genotypes Y62, Y66, Y67, S22, R35, W1, DAR12, L59 cultivated in vitro were shown in Figure 7c. Analysis of these data revealed a different $I_{i}^{q}$ profile than one shown in Figure 7a. $I^{q}{ }_{i}$ negative values had genotypes Y62, W1 and L59. This means that differences at the nucleotide sequences level influenced the auxin metabolism-related indices of genotypes otherwise than differences at the chromosomal (genome size) level. The comparison of two UQLI data series of eight genotypes for variants with $l_{1}=3$ and $l_{2}=3$ showed no linear dependence with a correlation coefficient $\mathrm{R}=0.3$ 
$\left(\mathrm{F}_{1,6}=0.582\right.$ compared to the tabular value of $5 \%$ limits for $\mathrm{N}=8 \mathrm{~F}_{1,6}=5.99$ ). Then UQLI was determined based on pairs of indices $\Delta \mathrm{Ph}_{\mathrm{i}}$ versus $\left|\Delta \mathrm{Gs}_{\mathrm{i}}\right|$, $\left|\Delta \mathrm{Fl}_{\mathrm{i}}\right|$ versus $\left|\Delta \mathrm{Gs}_{\mathrm{i}}\right|,\left|\Delta \mathrm{Fl}_{\mathrm{i}}\right|$ versus $\Delta \mathrm{Ph}_{\mathrm{i}},\left|\Delta \mathrm{Glg}_{\mathrm{i}}\right|$ versus $\Delta \mathrm{Ph}_{\mathrm{i}},\left|\Delta \mathrm{Glg}_{\mathrm{i}}\right|$ versus $\left|\Delta \mathrm{Fl}_{\mathrm{i}}\right|,\left|\Delta \mathrm{Glg}_{\mathrm{i}}\right|$ versus $\left|\Delta \mathrm{Gs}_{\mathrm{i}}\right|$ to understand how differences at both chromosomal and nucleoid sequences levels influenced to UQLI (Table 8, Fig. 7d).

The values of UQLI $\left(I_{i}^{q}\right)$ for eight genotypes samples cultivated in vitro Y62, Y66, Y67, S22, R35, W1, DAR12, L59 are shown in Figure 7d. The data analysis presented in this figure showed that in addition to Y66 and DAR12 (Fig. 7a), negative values also had W1 and L59 (Fig. 7d). It was revealed that comparison of eight genotypes UQLI data two series for variants with $l_{1}=3$ and $l_{2}=4$ (was provided by added data block $\left.\left|\Delta \mathrm{Glg}_{\mathrm{i}}\right|\right)$ showed a linear relationship with a correlation coefficient $\mathrm{R}=0.93\left(\mathrm{~F}_{1,6}=41.28\right.$ compared to the tabular value of $5 \%$ limits for $\mathrm{N}=8 \mathrm{~F}_{1,6}=5.99$ ).

This result can be interpreted as a generally synchronous $\left|\Delta \mathrm{Glg}_{\mathrm{i}}\right|$ and $\left|\Delta \mathrm{Gs}_{\mathrm{i}}\right|$ influence on $I^{q}{ }_{i}$ with a shift in the region of negative UQLI values of two more genotypes W1 and L59. To understand this result, we considered the genotypes correlation models. As expected, the influence of differences in such two indices as genetic distances $\left|\Delta \mathrm{Glg}_{\mathrm{i}}\right|$ and differences in genome size $\left|\Delta \mathrm{Gs}_{\mathrm{i}}\right|$ for such auxin metabolism-related indices as differences in leaf length $\Delta \mathrm{Ph}_{\mathrm{i}}$ and flavonoid content $\left|\Delta \mathrm{Fl}_{\mathrm{i}}\right|$ was different for genotypes with both different and the same origin. Correlation models of genotypes were shown in Figures 8, 9.

To take into account differences not only at the genome size but also at the pigment level, we formed not only a set of $\Delta \mathrm{Ph}_{\mathrm{i}}$ versus $\left|\Delta \mathrm{Gs}_{\mathrm{i}}\right|,\left|\Delta \mathrm{Fl}_{\mathrm{i}}\right|$ versus $\left|\Delta \mathrm{Gs}_{\mathrm{i}}\right|,\left|\Delta \mathrm{Fl}_{\mathrm{i}}\right|$ versus $\Delta \mathrm{Ph}_{\mathrm{i}}\left(l_{1}=3\right)$ but also the set $\left|\Delta \mathrm{Pg}_{\mathrm{ij}}\right|$ versus $\Delta \mathrm{Ph}_{\mathrm{i}}$, $\left|\Delta \operatorname{Pg}_{\mathrm{ij}}\right|$ versus $\left|\Delta \mathrm{Fl}_{\mathrm{i}}\right|,\left|\Delta \mathrm{Fl}_{\mathrm{i}}\right|$ versus $\Delta \mathrm{Ph}_{\mathrm{i}}\left(l_{2}=3\right)$ where $\left|\Delta \mathrm{Pg}_{\mathrm{ij}}\right|$ were chlorophyll A (ChlA, $j=1)$, chlorophyll B (ChlB, $j=2$ ), carotenoids (Car, $j=3$ ) in eleven studied D. antarctica genotypes (Table 9, Fig. 7e).

An example of UQLI $\left(I_{i}^{q}\right)$ for samples of 11 genotypes cultivated in vitro was shown in Figures $7 \mathrm{e}, \mathrm{f}$. It is interesting that the comparison of two series of data UQLI $\left(I_{i}^{q}\right) 11$ genotypes for variants with $l_{1}=3$ and $l_{3}=4$ (by addition of the data block $\mid \Delta \mathrm{Pg}_{\mathrm{ij}}$ ) has shown a correlation with $\mathrm{R}=0.924\left(\mathrm{~F}_{1,9}=52.767\right.$ compared to the tabular value of $5 \%$ limits for $\mathrm{N}=11 \mathrm{~F}_{1,9}=5.12$ ). This means that the UQLI $\left(I_{i}^{q}\right)$ profile when adding a data block $\left|\Delta \mathrm{Pg}_{\mathrm{ij}}\right|$ was conserved very well (correlation is statistical confidently), but $I^{q}{ }_{i}$ changed its sign for W1 and became zero for L59. Such influence of add-

Table 8. The United Latent Index of Adaptability calculation for the $i$-th genotype Deschampsia antarctica for value $I_{i}^{q}\left(l_{2}=4\right)$ by indices pairs $\Delta \mathrm{Ph}_{\mathrm{i}}$ versus $\left|\Delta \mathrm{Gs}_{\mathrm{i}}\right|\left(I_{i 1}\right),\left|\Delta \mathrm{Fl}_{\mathrm{i}}\right|$ versus $\left|\Delta \mathrm{Gs}_{\mathrm{i}}\right|\left(I_{i 2}\right),\left|\Delta \mathrm{Fl}_{\mathrm{i}}\right| \operatorname{versus} \Delta \mathrm{Ph}_{\mathrm{i}}\left(I_{i 3}\right),\left|\Delta \mathrm{Glg}_{\mathrm{i}}\right|$ versus $\Delta \mathrm{Ph}_{\mathrm{i}}\left(I_{i 4}\right),\left|\Delta \mathrm{Glg}_{\mathrm{i}}\right|$ versus $\left|\Delta \mathrm{Fl}_{\mathrm{i}}\right|\left(I_{i 5}\right),\left|\Delta \mathrm{Glg}_{\mathrm{i}}\right|$ versus $\left|\Delta \mathrm{Gs}_{\mathrm{i}}\right|\left(I_{i 6}\right)$ where $\left|\Delta \mathrm{Gs}_{\mathrm{i}}\right|$ are genome size differences, $\Delta \mathrm{Ph}_{\mathrm{i}}$ are leaf length differences, $\left|\Delta \mathrm{Fl}_{\mathrm{i}}\right|$ are flavonoid content in leaves differences and $\left|\Delta \mathrm{G} \mathrm{g}_{\mathrm{i}}\right|$ are genetic distances according to Jacquard

\begin{tabular}{|r|c|c|c|c|c|c|c|c|c|}
\hline $\mathrm{i}$ & Genotype name & $I_{i 1}$ & $I_{i 2}$ & $I_{i 3}$ & $I_{i}^{q}\left(l_{1}=3\right)$ & $I_{i 4}$ & $I_{i 5}$ & $I_{i 6}$ & $I_{i}^{q}\left(l_{2}=4\right)$ \\
\hline 1 & G/D4-1 & 0.8 & 0.4 & 0.2 & 0.466 & - & - & - & - \\
2 & G/D12-2a & 0.8 & 0.4 & 0.6 & 0.599 & - & - & - & - \\
3 & G/D12-1 & 0.8 & 0.4 & 0.6 & 0.599 & - & - & - & - \\
4 & Y62 & 0.4 & 0.2 & 0.0 & 0.200 & -0.33 & 0.11 & 0.33 & 0.119 \\
5 & Y66 & -0.2 & -0.4 & 0.0 & -0.200 & 0.11 & 0.33 & -0.56 & -0.120 \\
6 & Y67 & 0.6 & 0.6 & 0.2 & 0.466 & -0.33 & 0.33 & 0.56 & 0.327 \\
7 & S22 & 0.6 & 0.4 & 0.6 & 0.533 & 0.33 & 0.11 & 0.33 & 0.396 \\
8 & R35 & 0.6 & 0.4 & 0.0 & 0.333 & 0.11 & 0.33 & 0.11 & 0.259 \\
9 & W1 & 0.6 & 0.2 & -0.4 & 0.133 & -0.11 & -0.33 & -0.33 & -0.062 \\
10 & DAR12 & -0.2 & -0.4 & 0.0 & -0.200 & 0.11 & 0.11 & -0.11 & -0.082 \\
11 & L59 & 0.6 & -0.4 & 0.0 & 0.067 & 0.11 & -0.56 & -0.11 & -0.060 \\
\hline
\end{tabular}


ing of $\left|\Delta \operatorname{Pg}_{\mathrm{ij}}\right|$ set data to $I_{i}^{q}\left(l_{1}=3\right)$ resembles the influence adding of $\left|\Delta \mathrm{Glg}_{\mathrm{j}}\right|$ to the same index (Figs. 7d, f).

UQLI $\left(I_{i}^{q}\right)$ value was determined for eight genotypes taking into account the index components that influenced the sign of index, namely the data sets $\mathrm{Gs}_{\mathrm{i}}$, $\mathrm{Glg}_{\mathrm{i}}, \mathrm{Ph}_{\mathrm{i}}, \mathrm{Fl}_{\mathrm{i}}, \mathrm{Pg}_{\mathrm{i}}(\mathrm{l}=5)$ (Table 10).
The results presented in Table 10 indicated a significant influence of differences sets $\left|\Delta \mathrm{Glg}_{\mathrm{j}}\right|$ and $\left|\Delta \mathrm{Pg}_{\mathrm{ij}}\right|$ on UQLI $\left(I^{q}\right)$ sign of values at least for two genotypes $\mathrm{W} 1$ and $\operatorname{L59}(i=9, i=11)$. This means that $\left|\Delta \mathrm{Glg}_{\mathrm{i}}\right|$ and $\left|\Delta \mathrm{Pg}_{\mathrm{ij}}\right|$ influenced UQLI $\left(I_{i}^{q}\right)$ in the same phase.

Table 9. The United Latent Index of Adaptability calculation for the $i$-th genotype Deschampsia antarctica for value $I_{i}^{q}\left(l_{3}=4\right)$ by indices pairs $\Delta \mathrm{Ph}_{\mathrm{i}}$ versus $\left|\Delta \mathrm{Gs}_{\mathrm{i}}\right|\left(I_{i i}\right),\left|\Delta \mathrm{Fl}_{\mathrm{i}}\right|$ versus $\left|\Delta \mathrm{Gs}_{\mathrm{i}}\right|\left(I_{i 2}\right),\left|\Delta \mathrm{Fl}_{\mathrm{i}}\right|$ versus $\Delta \mathrm{Ph}_{\mathrm{i}}\left(I_{i 3}\right),\left|\Delta \mathrm{Pg}_{\mathrm{ij}}\right| \operatorname{versus} \Delta \mathrm{Ph}_{\mathrm{i}}$ $\left(I_{i 4}\right),\left|\Delta \mathrm{Pg}_{\mathrm{ij}}\right|$ versus $\left|\Delta \mathrm{Fl}_{\mathrm{j}}\right|\left(I_{i 5}\right),\left|\Delta \mathrm{Pg}_{\mathrm{ij}}\right|$ versus $\left|\Delta \mathrm{Gs}_{\mathrm{i}}\right|\left(I_{i 6}\right)$ where $\left|\Delta \mathrm{Gs}_{\mathrm{i}}\right|$ are genome size differences, $\Delta \mathrm{Ph}_{\mathrm{i}}$ are leaf length differences, $\left|\Delta \mathrm{Fl}_{\mathrm{i}}\right|$ are flavonoid content in leaves differences and $\left|\Delta \mathrm{Pg}_{\mathrm{ij}}\right|$ are photosynthetic pigments content differences

\begin{tabular}{|c|c|c|c|c|c|c|c|c|c|}
\hline $\mathrm{i}$ & Genotype name & $I_{i 1}$ & $I_{i 2}$ & $I_{i 3}$ & $I_{i}^{q}\left(l_{1}=3\right)$ & $I_{i 4}$ & $I_{i 5}$ & $I_{i 6}$ & $I_{i}^{q}\left(l_{3}=4\right)$ \\
\hline 1 & G/D4-1 & 0.8 & 0.4 & 0.2 & 0.466 & 0.133 & 0.6 & -0.067 & 0.345 \\
2 & G/D12-2a & 0.8 & 0.4 & 0.6 & 0.599 & -0.400 & -0.267 & -0.267 & 0.145 \\
3 & G/D12-1 & 0.8 & 0.4 & 0.6 & 0.599 & 0.133 & 0.067 & 0.133 & 0.356 \\
4 & Y62 & 0.4 & 0.2 & 0.0 & 0.200 & 0.000 & -0.067 & -0.067 & 0.078 \\
5 & Y66 & -0.2 & -0.4 & 0.0 & -0.200 & -0.400 & 0.067 & -0.267 & -0.200 \\
6 & Y67 & 0.6 & 0.6 & 0.2 & 0.466 & 0.000. & 0.067 & -0.133 & 0.223 \\
7 & S22 & 0.6 & 0.4 & 0.6 & 0.533 & 0.067 & -0.067 & -0.133 & 0.245 \\
8 & R35 & 0.6 & 0.4 & 0.0 & 0.333 & 0.067 & 0.333 & 0.00 & 0.234 \\
9 & W1 & 0.6 & 0.2 & -0.4 & 0.133 & 0.000 & -0.333 & -0.133 & -0.011 \\
10 & DAR12 & -0.2 & -0.4 & 0.0 & -0.200 & 0.133 & -0.200 & -0.333 & -0.167 \\
11 & L59 & 0.6 & -0.4 & 0.0 & 0.067 & -0.133 & 0.267 & -0.333 & 0.000 \\
\hline
\end{tabular}

Table 10. The United Latent Index of Adaptability calculation for the $i$-th genotype Deschampsia antarctica for value $I_{i}{ }_{i}\left(l_{2}=4\right)$ by indices pairs $\Delta \mathrm{Ph}_{\mathrm{i}}$ versus $\left|\Delta \mathrm{Gs}_{\mathrm{i}}\right|\left(I_{i 1}\right),\left|\Delta \mathrm{Fl}_{\mathrm{i}}\right|$ versus $\left|\Delta \mathrm{Gs}_{\mathrm{i}}\right|\left(I_{i 2}\right),\left|\Delta \mathrm{Fl}_{\mathrm{i}}\right|$ versus $\Delta \mathrm{Ph}_{\mathrm{i}}\left(I_{i 3}\right),\left|\Delta \mathrm{Glg}_{\mathrm{i}}\right|$ versus $\Delta \mathrm{Ph}_{\mathrm{i}}$ $\left(I_{i 4}\right),\left|\Delta \mathrm{Glg}_{\mathrm{j}}\right|$ versus $\left|\Delta \mathrm{Fl}_{\mathrm{i}}\right|\left(I_{i j}\right),\left|\Delta \mathrm{Glg}_{\mathrm{i}}\right|$ versus $\left|\Delta \mathrm{Gs}_{\mathrm{i}}\right|\left(I_{i 6}\right),\left|\Delta \mathrm{Pg}_{\mathrm{jij}}\right|$ versus $\Delta \mathrm{Ph}_{\mathrm{i}}\left(I_{i 7}\right),\left|\Delta \operatorname{Pg}_{\mathrm{ij}}\right|$ versus $\left|\Delta \mathrm{Fl}_{\mathrm{i}}\right|\left(I_{i 8}\right),\left|\Delta \operatorname{Pg}_{\mathrm{ij}}\right| \operatorname{versus}\left|\Delta \mathrm{Gs}_{\mathrm{i}}\right|\left(I_{i 9}\right)$, $\left|\Delta \mathrm{Glg} \mathrm{g}_{\mathrm{i}}\right|$ versus $\left|\Delta \mathrm{Pg}_{\mathrm{i}}\right|\left(I_{i 10}\right)$ where $\left|\Delta \mathrm{Gs}_{\mathrm{i}}\right|$ are genome size differences, $\Delta \mathrm{Ph}_{\mathrm{i}}$ are leaf length differences, $\left|\Delta \mathrm{Fl} \mathrm{i}_{\mathrm{i}}\right|$ are flavonoid content in leaves differences and $\left|\Delta \mathrm{Glg}_{\mathrm{i}}\right|$ are genetic distances according to Jacquard, $\left|\Delta \mathrm{Pg}_{\mathrm{ij}}\right|$ are photosynthetic pigments content differences

\begin{tabular}{|c|c|c|c|c|c|c|c|c|c|c|c|c|}
\hline $\mathrm{i}$ & Genotype name & $I_{i I}$ & $I_{i 2}$ & $I_{i 3}$ & $I^{q}{ }_{i 4}$ & $I_{i 5}$ & $I_{i 6}$ & $I_{i 7}$ & $I_{i 8}$ & $I_{i 9}$ & $I_{i l 0}$ & $I_{i}^{q}(l=5)$ \\
\hline 1 & G/D4-1 & 0.8 & 0.4 & 0.2 & - & - & - & 0.133 & 0.600 & -0.067 & - & - \\
2 & G/D12-2a & 0.8 & 0.4 & 0.6 & - & - & - & -0.4 & -0.267 & -0.267 & - & - \\
3 & G/D12-1 & 0.8 & 0.4 & 0.6 & - & - & - & 0.133 & 0.067 & 0.133 & - & - \\
4 & Y62 & 0.4 & 0.2 & 0.0 & -0.33 & 0.11 & 0.33 & 0.000 & -0.067 & -0.067 & 0.33 & 0.05840 \\
5 & Y66 & -0.2 & -0.4 & 0.0 & 0.11 & 0.33 & -0.56 & -0.400 & 0.067 & -0.267 & -0.56 & -0.1682 \\
6 & Y67 & 0.6 & 0.6 & 0.2 & -0.33 & 0.33 & 0.56 & 0.000 & 0.067 & -0.133 & 0.56 & 0.2066 \\
7 & S22 & 0.6 & 0.4 & 0.6 & 0.33 & 0.11 & 0.33 & 0.067 & -0.067 & -0.133 & 0.33 & 0.2342 \\
8 & R35 & 0.6 & 0.4 & 0.0 & 0.11 & 0.33 & 0.11 & 0.067 & 0.333 & 0.000 & 0.11 & 0.2303 \\
9 & W1 & 0.6 & 0.2 & -0.4 & -0.11 & -0.33 & -0.33 & 0.000 & -0.333 & -0.133 & -0.33 & -0.1064 \\
10 & DAR12 & -0.2 & -0.4 & 0.0 & 0.11 & 0.11 & -0.11 & 0.133 & -0.200 & -0.333 & -0.11 & -0.1243 \\
11 & L59 & 0.6 & -0.4 & 0.0 & 0.11 & -0.56 & -0.11 & 0.133 & 0.267 & -0.333 & -0.11 & -0.0616 \\
\hline
\end{tabular}



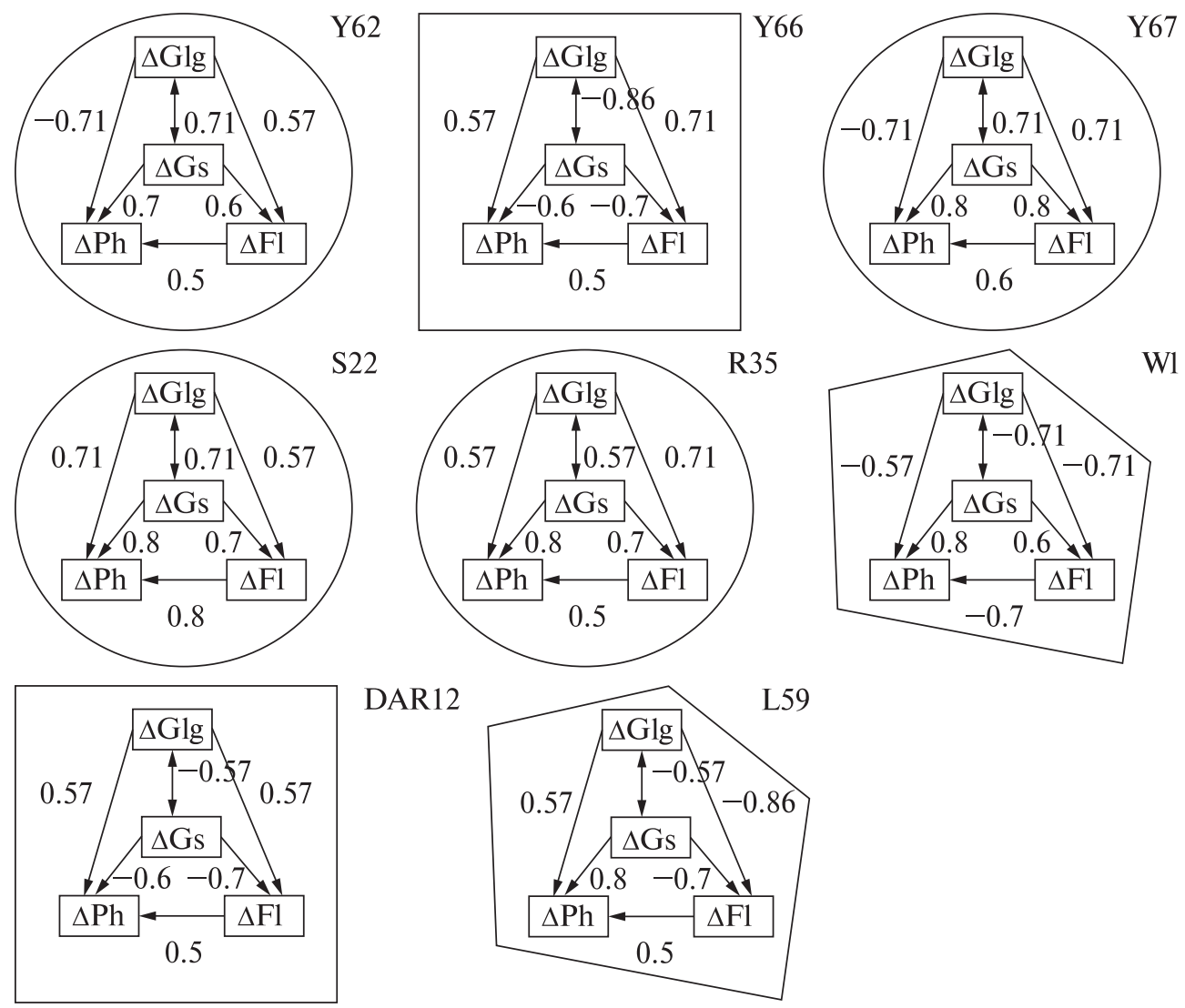

DAR12

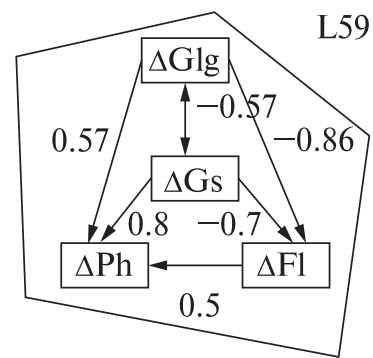

Figure 9. The bipartite graph of two probabilistic relations graphs $\left|\Delta \mathrm{Glg}_{\mathrm{i}}\right| \rightarrow \Delta \mathrm{Ph}_{\mathrm{i}}\left|\Delta \mathrm{Glg}_{\mathrm{i}}\right| \rightarrow\left|\Delta \mathrm{Fl}_{\mathrm{i}}\right|,\left|\Delta \mathrm{Fl}_{\mathrm{i}}\right| \rightarrow \Delta \mathrm{Ph}_{\mathrm{i}}$ and $\left|\Delta \mathrm{Gs}_{\mathrm{i}}\right| \rightarrow \Delta \mathrm{Ph}_{\mathrm{i}}$, $\left|\Delta \mathrm{Gs}_{\mathrm{i}}\right| \rightarrow\left|\Delta \mathrm{Fl}_{\mathrm{i}}\right|,\left|\Delta \mathrm{Fl}_{\mathrm{i}}\right| \rightarrow \Delta \mathrm{Ph}_{\mathrm{i}}$ ('genetic distances' graphs) and probabilistic connection $\left|\Delta \mathrm{Glg}_{\mathrm{i}}\right| \leftrightarrow\left|\Delta \mathrm{Gs}_{\mathrm{i}}\right|$ (connection of 'genetic distances' and 'genome size' graphs which provides certain relationships between graphs for the eight Deschampsia antarctica genotypes under in vitro condition based on four data sets $\left(l_{2}=4\right)$. The numbers indicate the probability of synchrony $(+)$ or asynchrony $(-)$ processes described by the compared data series for each genotype. The genotype graphs (2C, diploids) with most positive probability relations are outlined by ovals, graphs of genotypes with two negative relations $\left(\left|\Delta \mathrm{Gs}_{i}\right| \rightarrow \Delta \mathrm{Ph}_{\mathrm{i}},\left|\Delta \mathrm{Gs}_{\mathrm{i}}\right| \rightarrow\left|\Delta \mathrm{Fl}_{\mathrm{j}}\right|\right)$ are outlined by rectangles (there are 2C, diploid with B-chromosomes DAR12 and 3C, hypotriploid genotype Y66), 2C, diploid genotypes W1 and L59 with the most negative relations are outlined by pentagons. The pairwise differences by value $i$ where $i$ is genotype number (Table 1) are $\left|\Delta \mathrm{Gs}_{i}\right|$ for genome size, $|\Delta \mathrm{Glg}|$ for genetic distances, $\left|\Delta \mathrm{Fl}_{i}\right|$ for flavonoids content, $\Delta \mathrm{Ph}_{\mathrm{i}}$ for leaf length

Correlation models of the differences in genome size influence $\left(\left|\Delta \mathrm{Gs}_{\mathrm{i}}\right|\right)$ on differences in leaf length $\left(\Delta \mathrm{Ph}_{\mathrm{i}}\right)$ and flavonoid content $\left(\mid \Delta \mathrm{Fl}_{\mathrm{i}}\right)$ (auxin metabolism indices) for eleven genotypes represented by graphs of probability relations are shown in Figure 8.

Correlation models of the differences in genetic distances according to Jacquard $\left(\left|\Delta \mathrm{Glg}_{\mathrm{i}}\right|\right)$, which influenced auxin metabolism-related indices differences: in leaf length $\left(\Delta \mathrm{Ph}_{\mathrm{i}}\right)$ and flavonoid content $(|\Delta \mathrm{Fl}|)$ for eight genotypes are shown in Figure 9.

As can be seen from the graphs shown in Figure 9, we can distinguish two different graphs of base genetic characteristics and adaptation indices in the form of probabilistic relations between sets of pairwise distances. These were graphs of probabilistic relations $\left|\Delta \mathrm{Glg}_{\mathrm{i}}\right| \rightarrow \Delta \mathrm{Ph}_{\mathrm{i}},\left|\Delta \mathrm{Glg}_{\mathrm{i}}\right| \rightarrow\left|\Delta \mathrm{Fl}_{\mathrm{i}}\right|\left|\Delta \mathrm{Fl}_{\mathrm{i}}\right| \rightarrow \Delta \mathrm{Ph}_{\mathrm{i}}$ and $\left|\Delta \mathrm{Gs}_{\mathrm{i}}\right| \rightarrow \Delta \mathrm{Ph}_{\mathrm{i}},\left|\Delta \mathrm{Gs}_{\mathrm{i}}\right| \rightarrow\left|\Delta \mathrm{Fl}_{\mathrm{i}}\right|,\left|\Delta \mathrm{Fl}_{\mathrm{i}}\right| \rightarrow \Delta \mathrm{Ph}_{\mathrm{i}}$. We can single out the probabilistic relation $\left|\Delta \mathrm{Glg}_{\mathrm{i}}\right| \leftrightarrow\left|\Delta \mathrm{Gs}_{\mathrm{i}}\right|$ which characterizes the relationship between graphs.

To abbreviate, we introduce the terms 'genome size' graph and 'genetic distances' graph. The 'genome size' graph is the graph of the genome size differences (Fig. 8) influenced on auxin metabolism-related indices. In case genetic heterogeneity index (IRAP and 
ISSR marker combination) used the graph of differences in genetic distances between genotypes whose influence on auxin metabolism-related indices will be called 'genetic distances' graph. Its influence on 'genetic distances' graphs for eight genotypes is presented in Figure 9.

Analyzing genotype groups according to their correlation models represented by graphs of probability relations, we try to understand how the basic characteristics for each of them were realized under the new cultivation in vitro conditions. The basic genetic characteristics were saved in dynamic memory, which depended on the plant's origin according to our assumptions themselves based on Tchuraev (2006a).

1. Two genotypes of the same origin, Y62 and Y67 (2C, diploids), were included in the first group. All probabilistic relations in diploids in the inner graph ('genome size' graph path) were positive, and in the outer ('genetic distances' graph path) all ones except $\left|\Delta \mathrm{Glg}_{\mathrm{i}}\right|$ versus $\Delta \mathrm{Ph}_{\mathrm{i}}$ which means that increase/decrease in the value of the genetic distance $\left|\Delta \mathrm{Glg}_{\mathrm{j}}\right|$ accompanied by a decrease/ increase in $\Delta \mathrm{Ph}_{\mathrm{i}}$ values for Y62 and Y67 (Fig. 9). Probability graphs Y62 and Y67, which belong to the same group, can be interpreted as follows. The $\left|\Delta \mathrm{Glg}_{\mathrm{i}}\right|$ value between Y62 and Y67 was one of the smallest in this data set (0.0484), corresponding to a small value $\left|\Delta \mathrm{Fl}_{\mathrm{i}}\right|(1.02)$, which in turn corresponds to a small value of $\Delta \mathrm{Ph}_{\mathrm{i}}(0)$ (Table 4). This is the graph path' $\left|\Delta \mathrm{Glg}_{\mathrm{i}}\right| \rightarrow\left|\Delta \mathrm{Fl}_{\mathrm{i}}\right| \rightarrow \Delta \mathrm{Ph}_{\mathrm{i}}$. In this case, this path works in the 'genetic distances' graph' because the direct probabilistic connection $\left|\Delta \mathrm{Glg}_{\mathrm{i}}\right| \rightarrow$ $\rightarrow \Delta \mathrm{Ph}_{\mathrm{i}}$ contradicts such a schedule. The 'genome size' graph does not have such contradictions, so it is the main one for these two genotypes. This situation in genotypes Y62 and Y67 corresponds to a small average leaf length (Fig. 1), which does not differ for these two genotypes. Hence, this group of genotypes is stable under the chosen growing conditions.

2. The Y66 genotype graph which has the same origin as Y62 and Y67, but has a chromosomal polymorphism (hypotriploid according to Navrotska et al. (2014) can be attributed to the same group as another genotype with chromosomal polymorphism DAR12 (presence of B-chromosome (Amosova et al., 2015)) with a completely different origin. These two geno- types (the second group) have similar graphs of probabilistic relations in the studied data sets which are strikingly different in the 'genome size' graph $\left(\left|\Delta \mathrm{Gs}_{\mathrm{i}}\right| \rightarrow\right.$ $\left.\rightarrow \Delta \mathrm{Ph}_{\mathrm{i}},\left|\Delta \mathrm{Gs}_{\mathrm{i}}\right| \rightarrow\left|\Delta \mathrm{Fl}_{\mathrm{i}}\right|,\left|\Delta \mathrm{Fl}_{\mathrm{i}}\right| \rightarrow \Delta \mathrm{Ph}_{\mathrm{i}}\right)$ from genotypes with chromosomes diploid sets matched into the negative group by $I^{q}\left(l_{1}=3\right)$ in the case of only the 'genome size' graph (Figs. 7a, 8), by $I_{i}^{q}\left(l_{1}=4\right)$ in the case of the 'genome size' graph and the graph of 'protective proteins' $\left(\left|\Delta \mathrm{Gs}_{\mathrm{i}}\right| \rightarrow \Delta \mathrm{Ph}_{\mathrm{i}},\left|\Delta \mathrm{Gs}_{\mathrm{i}}\right| \rightarrow\left|\Delta \mathrm{Fl}_{\mathrm{i}}\right|,\left|\Delta \mathrm{Fl}_{\mathrm{i}}\right| \rightarrow\right.$ $\rightarrow \Delta \mathrm{Ph}_{\mathrm{i}},\left|\Delta \mathrm{Pr}_{\mathrm{ik}}\right| \rightarrow \Delta \mathrm{Ph}_{\mathrm{i}},\left|\Delta \mathrm{Pr}_{\mathrm{ik}}\right| \rightarrow\left|\Delta \mathrm{Fl}_{\mathrm{i}}\right|,\left|\Delta \mathrm{Gs}_{\mathrm{i}}\right| \rightarrow$ $\left.\rightarrow\left|\Delta \operatorname{Pr}_{\text {ik }}\right|\right)$ (Fig. 7b), according to $\mathrm{I}_{\mathrm{i}}^{\mathrm{q}}\left(l_{2}=4\right)$ in the case of taking into account the 'genome size' graph and the 'genetic distances' graph $\left(\left|\Delta \mathrm{Glg}_{\mathrm{i}}\right| \rightarrow \Delta \mathrm{Ph}_{\mathrm{i}},\left|\Delta \mathrm{Glg}_{\mathrm{i}}\right| \rightarrow\right.$ $\left.\left|\Delta \mathrm{Fl}_{\mathrm{i}}\right|,\left|\Delta \mathrm{Fl}_{\mathrm{i}}\right| \rightarrow \Delta \mathrm{Ph}_{\mathrm{i}}\right)$ (Fig. 7d). However, the Y66 genotype has more significant values $(0.57,0.71$ versus $0.57,0.57$, and -0.86 versus -0.57 ) in DAR12 probabilistic relationships in the 'genetic distances' graph and between the 'genome size' and 'genetic distances' graphs (Fig. 9). Probability graphs Y66 and DAR12, which we refer to as one group, can be interpreted as follows. The $\left|\Delta \mathrm{Glg}_{\mathrm{i}}\right|$ value between Y66 and DAR12 was twice as small as the maximum in this data set (0.0964). It corresponds to a small value $\left|\Delta \mathrm{Fl}_{\mathrm{i}}\right|(1.01)$, which, in turn, corresponds to one of the smallest $\Delta \mathrm{Ph}$ values (27.68) (Table 4). This was the path $\left|\Delta \mathrm{Glg}_{\mathrm{i}}\right| \rightarrow$ $\left|\Delta \mathrm{Fl}_{\mathrm{i}}\right| \rightarrow \Delta \mathrm{Ph}_{\mathrm{i}}$. In this case, the direct probability relationship $\left|\Delta \mathrm{Glg}_{\mathrm{i}}\right| \rightarrow \Delta \mathrm{Ph}_{\mathrm{i}}$ also worked harmoniously. Probabilistic relationship of 'genome size' and 'genetic distances' graphs $\left|\Delta \mathrm{Glg}_{\mathrm{i}}\right|(0.0968)$ corresponded to $\left|\Delta \mathrm{Gs}_{\mathrm{i}}\right|(5.88)$ and was negative $(-0.86$ and -0.57$)$ for Y66 and DAR12 genotypes correspondingly (Fig. 9). These genotypes had a much longer average leaf length (approximately 11 versus $5 \mathrm{~cm}$ in Y62 and Y67) and a large distribution range (Fig. 1). Both graphs worked harmoniously.

3. Genotypes S22 and R35 (third group) can be characterized by the presence of only positive and neutral relations in both graphs. The $\left|\Delta \mathrm{Glg}_{\mathrm{j}}\right|$ value between S22 and R35 was approximately twice less than the maximum in this data set (0.0953), it corresponded to a small value $\left|\Delta \mathrm{Fl}_{\mathrm{i}}\right|(1.02)$ which, in turn, is approximately three times less than the maximum in this data set $\Delta \mathrm{Ph}_{\mathrm{i}}$ (81.26) (Table 4). Hence the work of the 'genetic distances' graph did not go beyond harmonious work. The work of the 'genome size' graph $\left(\left|\Delta \mathrm{Gs}_{\mathrm{i}}\right|\right.$ 
(0.17), $\left.\left|\Delta \mathrm{Fl}_{\mathrm{i}}\right|(1.02), \Delta \mathrm{Ph}_{\mathrm{i}}(81.26)\right)$ was harmonious also. The probabilistic relationship between the graphs was positive. These genotypes had a slightly longer average leaf length than Y62 and Y67 (approximately $6-7 \mathrm{~cm})$. Among the differences should be noted the greater $\left|\Delta \mathrm{Fl}_{\mathrm{i}}\right|$ influence on $\Delta \mathrm{Ph}_{\mathrm{i}}$ in genotype S22, which had a shorter leaf length compared to R35 (0.8 versus 0.5 ) (Fig. 9).

4. The fourth group of genotypes W1 and L59 (diploids) was interesting because it had a positive and a negative relationship in the 'genome size' graph and had positive $I^{q}{ }_{i}\left(l_{1}=3\right)$ values under taking into account only the 'genome size' graph conditions. If the 'genome size' and 'genetic distances' graphs were taken into account the $I_{i}^{q}\left(l_{2}=4\right)$ values of these genotypes turn into a negative group. We suppose this probably means that the 'genetic distances' graph is more important for these genotypes. But since these two genotypes graphs differ from each other, we consider them separately. The $\left|\Delta \mathrm{Glg}_{\mathrm{j}}\right|(0.1639)$ value between W1 and L59 was one of the largest values in this data set; it corresponded to a large $\left|\Delta \mathrm{Fl}_{\mathrm{i}}\right|(2.44)$ value, which in turn corresponded to $\Delta \mathrm{Ph}_{\mathrm{i}}(14.03)$, one of the smallest in this data set, the $\left|\Delta \mathrm{Gs}_{\mathrm{i}}\right|(0.1)$ value was one of the smallest also (Table 4). For the W1 genotype, this means that three independent pathways, $\left|\Delta \mathrm{Glg}_{\mathrm{i}}\right| \rightarrow \Delta \mathrm{Ph}_{\mathrm{i}}$ in the 'genetic distances' graph, $\left|\Delta \mathrm{Gs}_{\mathrm{i}}\right|$ $\rightarrow \Delta \mathrm{Ph}_{\mathrm{i}}$ in the 'genome size' graph, and $\left|\Delta \mathrm{Fl}_{\mathrm{i}}\right| \rightarrow \Delta \mathrm{Ph}$ in both graphs influenced $\Delta \mathrm{Ph}_{\mathrm{i}} \cdot \Delta \mathrm{Glg}_{\mathrm{i}}|\rightarrow| \Delta \mathrm{Fl}_{\mathrm{i}} \mid$ path in the 'genetic distances' graph and $\left|\Delta \mathrm{Gs}_{\mathrm{i}}\right| \rightarrow\left|\Delta \mathrm{Fl}_{\mathrm{i}}\right|$ path in the 'genome size' graph should be acted in antiphase to $\Delta \mathrm{Ph}_{\mathrm{i}}$ with probabilities of 0.7 and 0.6 , respectively. It is possible that in this case, there were variants corresponding to probabilities 0.3 and 0.4 , respectively. However, both graphs acted in concert: $\left|\Delta \mathrm{Gs}_{\mathrm{i}}\right|$ and $\left|\Delta \mathrm{Glg}_{\mathrm{i}}\right|$ had a negative probability relationship (-0.71). For the L59 genotype, this meant that the $\left|\Delta \mathrm{Gs}_{\mathrm{i}}\right| \rightarrow \Delta \mathrm{Ph}_{\mathrm{i}}$ and $\left|\Delta \mathrm{Gs}_{\mathrm{i}}\right| \rightarrow\left|\Delta \mathrm{Fl}_{\mathrm{i}}\right|$ pathways acted in concert in the 'genome size' graph, leaving the path $\left|\Delta \mathrm{Fl}_{\mathrm{i}}\right| \rightarrow \Delta \mathrm{Ph}_{\mathrm{i}}$ neutral. The $\left|\Delta \mathrm{Glg}_{\mathrm{i}}\right| \rightarrow\left|\Delta \mathrm{Fl}_{\mathrm{i}}\right|(-0.86)$ and $\left|\Delta \mathrm{Glg}_{\mathrm{i}}\right| \rightarrow\left|\Delta \mathrm{Ph}_{\mathrm{i}}\right|(0.57)$ paths acted in antiphase in the 'genetic distances' graph. However, both graphs acted in concert because $\left|\Delta \mathrm{Gs}_{\mathrm{i}}\right| \rightarrow\left|\Delta \mathrm{Glg}_{\mathrm{i}}\right|$ path had a negative probability relation $(-0.57)$. So, both base genetic characteristicsgraphs were involved in the W1 genotype, and the 'genome size' graph predominated in L59. As a result, the average leaf length in W1 and L59 (approximately $7.5 \mathrm{~cm}$ ) differed little, but the distribution of this parameter in L59 had a larger swing to increasing leaf length; the flavonoid content was 2.59 in W1 and $4.67 \mathrm{mg} / \mathrm{g}$ in L59 genotypes.

Flavonoids are regulators of auxin metabolism; in particular, luteolin is a synergist of auxin, and apigenin inhibits the synthesis of IAA (indolyl-3-acetic acid) because it is a cofactor of IAA oxidase (Makarenko \& Levitsky, 2013). We did not isolate the content of luteolin and apigenin separately when measuring the total flavonoid content, although, according to our assumptions, the nature of the interaction of genetic distances with adaptability indices may reflect the regulation of their synthesis. Therefore, the discrepancy between $\left|\Delta \mathrm{Glg}_{\mathrm{i}}\right|$ and final $\left|\Delta \mathrm{Fl}_{\mathrm{i}}\right|$ values probabilistic connection on the path $\left|\Delta \mathrm{Glg}_{\mathrm{i}}\right| \rightarrow\left|\Delta \mathrm{Fl}_{\mathrm{i}}\right|$ may be caused by auxin synthesis stimulation or blocking in this path.

\section{Conclusions}

The developed algorithm for the UQLI calculation has been used to evaluate the complex adaptability for eleven genotypes of $D$. antarctica cultivated in vitro with different origins from sites of the Argentine Islands region, the maritime Antarctic.

The individuality of the adaptive portrait for all studied $D$. antarctica genotypes under in vitro cultivation conditions was shown. The possible influence of basic genetic characteristics genome size and genetic distances according to IRAP and ISSR markers on auxin metabolism-related 'leaf length' and 'flavonoid content' indices was shown. Such influence may be carried out by genetic characteristics individually as well as in complex. Among the eight genotypes researched, we distinguish four different variants by correlation models and two (positive and negative) by the general $I_{i}^{q}$ value.

Thus the $I^{q}{ }_{i}$ (UQLI) is proposed to describe a lot of source data at different organization levels that characterize sample genotypes of the same species from different regions by reducing the dimension to one dimensionless number. The $I_{i}^{q}$ (UQLI) can be used to compare 
a set of genotypes sample of the same species growing under different conditions, especially during the artificial processing of genotypes by different factors designed to improve productivity on a given index.

This genotype individuality and their grouping by $\mathrm{I}^{\mathrm{q}}$ peculiarities should be taken into account in experimental studies using these genotypes as model plants.

Author contributions. NM: conceptualization, statistical data processing, development of algorithm for calculation UQLI and calculation of correlation models. Writing - original \& draft. IP: conceptualization, collecting seeds in nature, writing - review \& editing. OP: germination of seeds, creation and maintenance of a collection of plants, plant morphometry, review of works on plant biochemistry. Writing - review \& editing. GM: Determination of flavonoid concentration in leaves, electrophoresis of proteins in plant leaves, plant morphometry. Writing - review \& editing. MR-Je: determination of genome size in plant leaves. Writing - review \& editing. ED: general management of research by National Antarctic Scientific Center. Writing - review \& editing. VK: general management of research by Institute of Molecular Biology and Genetics. Writing - review \& editing.

Acknowledgments. This work was supported by the State Institution National Antarctic Scientific Center, Ministry of Education and Science of Ukraine, according to the State Special-Purpose Research Program in Antarctica for 2011-2020. We are grateful to Dr. I. Kozeretska and Prof. R Hasterok for assistance in organizing this study, and to reviewers, whose valuable comments and suggestions improved the presented paper.

Conflict of Interest. The authors declare that they have no conflict of interest.

\section{References}

Amosova, A. V., Bolsheva, N. L., Samatadze, T. E., Twardovska, M. O., Zoshchuk, S. A., Andreev, I. O., Badaeva, E. D., Kunakh, V. A., \& Muravenko, O. V. (2015). Molecular cytogenetic analysis of Deschampsia antarctica Desv. (Poaceae), Maritime Antarctic. PLoS ONE, 10(9), e0138878. https://doi. org/10.1371/journal.pone.0138878

Andreev, I. O., Spiridonova, E. V., Kyryachenko, S. S., Parnikoza, I. Yu., Maidanyuk, D. N., Volkov, R. A., Kozeretska,
I. A., \& Kunakh, V. A. (2010). Population-genetic analysis of Deschampsia antarctica from two regions of maritime antarctica. Moscow University Biological Sciences Bulletin, 65(4), 208-210.

Ayvazyan, S. A., Buchstaber, V. M., Yenyukov, I. S., \& Meshalkin, L. D. (1989). Prikladnaja statistika. Klassifikacija $i$ snizhenie razmernosti [Applied statistics. Classification and dimensionality reduction]. Finansy i statistika. (in Russian)

Bai, C., Alverson, W. S., Follansbee, A., \& Waller, D. M. (2012). New reports of nuclear DNA content for 407 vascular plant taxa from the United States. Annals of Botany, 110(8), 1623-1629. https://doi.org/10.1093/aob/mcs222

Bauman, E. V., \& Moskalenko, N. E. (2008). Methods of extremal grouping of the fractional parameters. Automation and Remote Control, 69, 1965-1972. https://doi.org/10.1134/ S0005117908110131

Corder, G. W., \& Foreman, D. I. (2014). Nonparametric Statistics: A Step-by-Step Approach. (2nd ed.). Wiley.

Ermakov, A. I.(Ed.). (1987). Metody biokhimicheskogo issledovanija rastenij [Methods of biochemical research of plants]. Agropromizdat. (in Russian)

Gill, S. S., \& Tuteja, N. (2010). Reactive oxygen species and antioxidant machinery in abiotic stress tolerance in crop plants. Plant Physiology and Biochemistry, 48(12), 909-930. https://doi.org/10.1016/j.plaphy.2010.08.016

Grotewold, E. (Ed.). (2006). The Science of flavonoids. Springer Science.

Iordachescu, M., \& Imai, R. (2008). Trehalose biosynthesis in response to abiotic stresses. Journal of Integrative Plant Biology, 50(10), 1223-1229. https://doi.org/10.1111/j.17447909.2008.00736.x

Kreps, J. A., Wu, Y., Chang, H.-S., Zhu, T., Wang, X., \& Harper, J. F. (2002). Transcriptome changes for Arabidopsis in response to salt, osmotic, and cold stress. Plant Physiology, 130(4), 2129-2141. https://doi.org/10.1104/pp.008532

Lambers, H., Chapin, III S. F., \& Pons, T. L. (2008). Plant Physiological Ecology. Springer-Verlag, New York.

Lee, J., Noh, E. K., Choi, H.-S., Shin, S. C., Park, H., \& Lee, H. (2012). Transcriptome sequencing of the Antarctic vascular plant Deschampsia Antarctica Desv. under abiotic stress. Planta, 237(3), 823-836. https://doi.org/10.1007/s00425-012$1797-5$

Makarenko, O. A., \& Levitsky, A. P. (2013). Physiological functions of flavonoids in plants. Physiology and biochemistry of cultivated plants, 45(2), 100-112. (in Russian)

Miryuta, N., Poronnik, O., Parnikoza, I., Grahov, V., Myryuta, A., Kozub, N., Sozinov, I., \& Kunakh, V. (2016). Conservation of complex adaptability uniqueness in different Deschampsia antarctica Desv. plant genotypes under standardized growth condition in vitro. Ukrainian Antarctic Journal, 15, 60-80. (in Ukrainian)

Miryuta, N. Yu., Parnikoza, I. Yu., Poronnik, O. O., Myryuta, G. Yu., \& Kunakh, V. A. (2017). Deschampsia antarctica 
E. Desv. with different chromosome numbers cultivated in vitro. Probabilistic relations of three adaptability indices with genome size. Factors of experimental evolution of organisms, 20, 293-298. (in Ukrainian)

Miryuta, N., Smykla, J., \& Parnikoza, I. (2019a). Algorithm for the United Quality Latent Index of the plant adaptability and its application field in monitoring of Deschampsia antarctica È. Desv. populations. Ukrainian Antarctic Journal, 1(18), 152-168. http://dspace.nbuv.gov.ua/bitstream/handle/ 123456789/168303/12-Miryuta.pdf?sequence=1

Miryuta, N., Wojciechowski, K., \& Parnikoza, I. (2019b). Calculation of the external factors influence indices on plants and its application to Deschampsia antarctica $\dot{\mathrm{E}}$. Desv. populations. Ukrainian Antarctic Journal, 2(19), 97-116.

Nakashima, K., Ito, Y., \& Yamaguchi-Shinozaki, K. (2009). Transcriptional regulatory networks in response to abiotic stresses in Arabidopsis and Grasses. Plant Physiology, 149(1), 88-95. https://doi.org/10.1104/pp.108.129791

Navrotska, D. O., Twardowska, M. O., Andreev, I. O., Parnikoza, I. Yu., Betekhtin, A. A., Zahrychuk, O. M., Drobyk, N. M., Hasterok, R., \& Kunakh, V. A. (2014). New forms of chromosome polymorphism in Deschampsia antarctica Desv. from the Argentine Islands of the Maritime Antarctic region. Ukrainian Antarctic Journal, 13, 185-191.

Navrotska, D. O., Andreev, I. O., Parnikoza, I. Yu., Spiridonova, K. V., Poronnik, O. O., Miryuta, N. Yu., Myryuta, G. Yu., Zahrychuk, O. M., Drobyk, N. M., \& Kunakh, V. A. (2017). Comprehensive characterization of cultivated in vitro Deschampsia antarctica E. Desv. plants with different chromosome numbers. Cytology and Genetics, 51(6), 422-431. https://doi.org/10.3103/S009545271706010X

Parnikoza, I., Miryuta, N., Ozheredova, I., Kozeretska, I., Smykla, J., Kunakh, V., \& Convey, P. (2015). Comparative analysis of Deschampsia antarctica Desv. population adaptability in the natural environment of the Admiralty Bay region (King George Island, maritime Antarctic). Polar Biology, 38(9), 14011411. https://doi.org/10.1007/s00300-015-1704-1

Parnikoza, I. Yu., Miryuta, N. Yu., Royek, M., Betekhtin, A. A., Poronnik, O. O., Miryuta, G. Yu., Navrotska, D. O., Hasterok, R., \& Kunakh, V. A. (2017). Deschampsia antarctica E. Desv. plants with different chromosome number cultivated in vitro. Relations between genome size and two adaptability indices. Factors of experimental evolution of organisms, 20, 304-309. (in Ukrainian)

Pollard, J. H. P. (1982). Spravochnik po vychislitelnym metodam statistiki [A handbook of numerical and statistical techniques]. Finansy i statistika. (in Russian)

Poronnik, O. O., Parnikoza, I. Yu., Miryuta, N. Yu., Myryuta, G. Yu., Grahov, V. P., Navrotska, D. O., \& Kunakh, V. A. (2017). Deschampsia antarctica E. Desv. plants with different chromosome number cultivated in vitro. Plants length and fla- vonoids in vitro culture and in nature. Factors of experimental evolution of organisms, 20, 310-313. (in Ukrainian)

Poronnik, O., Miryuta, N., Ivannikov, R., Myiryuta, G., Korchevska, V., Parnikoza, I., \& Kunakh, V. (2019, May 14-16). Long-term action of ultraphiolet an influence on different adaptability parameters of Deshampsia antarctica É. Desv. plants under cultivation in vitro [Conference presentation abstract]. IX International Antarctic Conference, Kyiv, Ukraine.

Scion Image: http://scion-image.software.informer.com/4.0/

Shalygo, N. V., Domanskaya, I. N., Radyuk, M. S., Shcherbakov, R. A., \& Dremuk, I. A. (2012). Accumulation of hydrogen peroxide and functioning of defense system in overwatered barley seedlings. Russian Journal of Plant Physiology, 59(6), 748-756. https://doi.org/10.1134/S1021443712050147 (in Russian)

Shinozaki, K., Yamaguchi-Shinozaki, K., \& Seki, M. (2003). Regulatory network of gene expression in the drought and cold stress responses. Current Opinion in Plant Biology, 6(5), 410-417. https://doi.org/10.1016/s1369-5266(03)00092-X

Tchuraev, R. N. (2006a). Epigenetics: gene and epigene networks in ontogeny and phylogeny. Genetika, 42(9), 12761296. (in Russian)

Tchuraev, R. N. (2006b). General principles of organization and laws of functioning in governing gene networks. In N. Kolchanov, R. Hofestädt, Milanesi, L. (Eds.), Bioinformatics of Genome Regulation and Structure II (pp. 367-377). Springer, Science Media Inc.

Van Loon, L. C. (Ed.) (2009). Plant Innate Immunity (Vol. 51). Academic Press.

Volkov, R. A., Kozeretska, I. A., Kyryachenko, S. S., Andreev, I. O., Maidanyuk, D. N., Parnikoza, I. Yu., \& Kunakh, V. A. (2010). Molecular evolution and variability of ITS1-ITS2 in populations of Deschampsia antarctica from two regions of the maritime Antarctic. Polar Science, 4(3), 469-478. https://doi. org/10.1016/j.polar.2010.04.011

Zahrychuk, O. M., Drobyk, N. M., Kozeretska, I. A., Parnikoza, I. Yu., \& Kunakh, V. A. (2011-2012). Introduction in culture in vitro of Deschampsia antarctica Desv. (Poaceae) from two regions of Maritime Antarctica. Ukrainian Antarctic Journal, 10-11, 289-295. (in Ukrainian)

Zubairova, U. S., Penenko, A. V., \& Nikolaev, S. V. (2012). Modeling of plant tissue growth and development with L-systems. Vavilov Journal of Genetics and Breeding, 16(4/1), 816824. (in Russian)

Zubairova, U. S., Golushko, S. K., Penenko, A. V., \& Nikolaev, S. V. (2014). An L-system for modeling flat unidimensionally growing flat plant tissues. Vavilov Journal of Genetics and Breeding, 18(4/2), 945-952. (in Russian)

Received: 16 November 2020 Accepted: 09 July 2021 


\section{Н. Мірюта ${ }^{1}$, I. Парнікоза ${ }^{1,2,3, *}$, О. Пороннік ${ }^{2,1}$, Г. Мирюта ${ }^{2,1}$,} М. Ройєк-Слонек ${ }^{4}$, Є. Дикий ${ }^{1}$, В. Кунах ${ }^{2}$

1 Державна установа Національний антарктичний науковий центр МОН України, м. Київ, 01601, Україна

${ }^{2}$ Інститут молекулярної біології і генетики НАН України, м. Київ, 03143, Україна

${ }^{3}$ Національний університет «Києво-Могилянська академія», м. Київ, 04655, Україна

${ }^{4}$ Сілезький Університет в Катовицях, м. Катовиці, 40-032, Польща

* Автор для кореспонденції: ivan.parnikoza@uac.gov.ua

Розрахунок зведеного латентного показника пристосовуваності генотипів Deschampsia antarctica різного походження вирощуваних in vitro

Реферат. Розроблено і детально описано алгоритм розрахунку зведеного латентного показника пристосовуваності $\left(I^{q}{ }^{2}\right.$, ЗЛПП) рослин із колекції генотипів Deschampsia antarctica $\dot{\mathrm{E}}$. Desv., отриманих з насіння з різних локалітетів регіону Аргентинських островів, морська Антарктика, і вирощуваних in vitro в лабораторних умовах. Як базові показники вихідної генетичної гетерогенності аналізованих культивованих генотипів рослин використано розмір генома (значення 2С ядерної ДНК для одинадцяти генотипів) та величини генетичних відстаней за ISSR та IRAP маркерами за даними, наведеними в опублікованій в роботі. Для оцінки окремих показників пристосовуваності для одинадцяти генотипів D. antarctica застосовано методи вимірювання морфометричного показника довжини листка, визначення кількості флавоноїдів за рутином та вмісту фотосинтетичних пігментів. Спектри запасних і захисних білків листків досліджено за допомогою електрофорезу в поліакриламідному гелі. Для отримання $I_{i}^{q}$ застосовано метод екстремального групування. Розрахунок ЗЛПП проводили за допомогою попарних порівнянь рядів різниць показників для кожної пари генотипів. Розроблено і детально описано алгоритм розрахунку $I^{q}{ }_{i}$ на прикладі одинадцяти генотипів D. antarctica. Як приклад застосування, наведено кореляційні моделі ймовірнісних відносин виміряних показників. Розроблений алгоритм розрахунку $I^{q}{ }_{i}$ було успішно використано для оцінки комплексної адаптованості одинадцяти генотипів D. antarctica, вирощуваних in vitro. Показано індивідуальність адаптаційного портрету усіх досліджуваних генотипів в умовах стандартизованого вирощування. Показано вплив основних генетичних характеристик: розміру генома та генетичних відстаней на пов'язані з ауксиновим метаболізмом показники пристосовуваності: довжину листків та вміст флавоноїдів. Серед восьми досліджуваних генотипів ми виділяємо чотири різні варіанти за кореляційними моделями та два (позитивні та негативні) за загальним $I^{q}{ }_{i}$.

Запропонований інтегральний показник ( $I^{q}$, ЗЛПП) може бути використаний для опису великої кількості вихідних даних, що характеризують генотипи в умовах вирощування in vitro на різних рівнях організації, за допомогою методів зниження розмірності, кінцевим результатом застосування яких є одне число без розмірності. Індивідуальність генотипів та їх групування за особливостями $I^{q}{ }_{i}$ слід враховувати під час проведення експериментальних досліджень із використанням цих генотипів як модельних рослин, особливо в дослідах з вивчення і регуляції продуктивності, вивчення впливу різних екзогенних чинників тощо.

Ключові слова: Deschampsia antarctica, культура рослин in vitro, зведений латентний показник пристосовуваності рослин, кореляційні моделі ймовірнісних відносин різних показників 